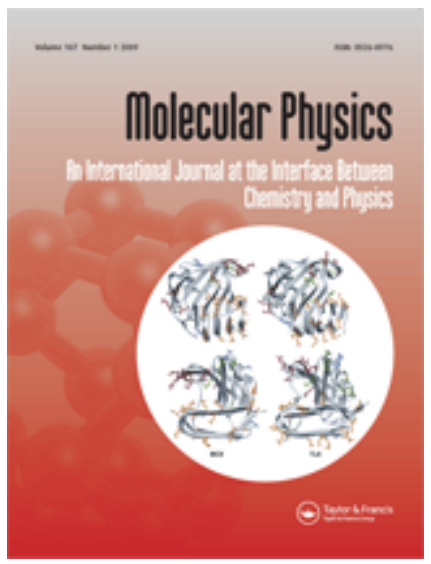

\title{
A Theoretical Investigation on the Oxidation States of Palladium Complexes and their Role in Carbonylation Reaction
}

\begin{tabular}{|c|c|}
\hline Journal: & Molecular Physics \\
\hline Manuscript ID: & TMPH-2009-0423.R1 \\
\hline Manuscript Type: & Full Paper \\
\hline $\begin{array}{r}\text { Date Submitted by the } \\
\text { Author: }\end{array}$ & 30-Mar-2010 \\
\hline Complete List of Authors: & $\begin{array}{l}\text { Miscione, Gian Pietro; University of Bologna, Dipartimento di } \\
\text { Chimica } \\
\text { Carvajal, Maria Angels; Universitat Rovira i Virgili, Departament de } \\
\text { Quimica Fisica i Inorganica } \\
\text { bottoni, andrea; University of Bologna, Department of Chemistry } \\
\text { Novoa, Juan; Universitat de Barcelona }\end{array}$ \\
\hline Keywords: & carbonylation, DFT, palladium, reaction mechanism, catalysis \\
\hline \multicolumn{2}{|c|}{$\begin{array}{l}\text { Note: The following files were submitted by the author for peer review, but cannot be converted } \\
\text { to PDF. You must view these files (e.g. movies) online. }\end{array}$} \\
\hline \multicolumn{2}{|l|}{$\begin{array}{l}\text { Schemes_Figures_Mol_Phys.zip } \\
\text { tables.zip }\end{array}$} \\
\hline
\end{tabular}

\section{今) ScholaroNE" Manuscript Central}




\title{
A Theoretical Investigation on the Oxidation States of Palladium Complexes and their Role in Carbonylation Reaction
}

\author{
Andrea Bottoni $^{(\mathrm{a})}$, Maria Angels Carvajal ${ }^{(\mathrm{b})}$, Gian Pietro Miscione $^{(\mathrm{a})}$,* \\ Juan J. Novoa ${ }^{(\mathrm{c})}$
}

(a) Dipartimento di Chimica "G. Ciamician", Universita' di Bologna, via Selmi 2, 40126 Bologna, Italy

(b) Departament de Quimica Fisica i Inorganica, Universitat Rovira i Virgili, Marcel.li Domingo, s/n-Campus Sescelades, 43007 Tarragona, Spain

(c) Departament de Quimica Fisica, Facultat de Quimica, Universitat de Barcelona, Av. Diagonal 647, 08028 Barcelona, Spain University, City, Country

Gian Pietro Miscione

Dipartimento di Chimica "G.Ciamician"

Via Selmi, 2 - 40126 Bologna, Italy

Tel. +39-051-2099524

Fax. + 39-051-2099456

Email: gianpietro.miscione@unibo.it

\begin{abstract}
Two different yet related topics are discussed: (i) the reduction of palladium (II) in $\mathrm{Pd}(\mathrm{OAc})_{2}$ complexes reacting with phenyl phosphines and leading to $\mathrm{Pd}(0)$ phosphines complexes and (ii) the carbonylation reaction of allyl chlorides catalyzed by these $\operatorname{Pd}(0)$ species. The results show that the overall reduction is an exothermic process that can be accomplished along two different reaction paths: one of them is clearly favoured.

Similarly, three different channels have been determined for the carbonylation reaction that primarily differ for the timing and the way with which the reacting species bind the metal. In the first path ( $\sigma$-path), the allyl fragment interacts very weakly with the metal, whereas, successively the $\mathrm{CO}$ molecule strongly binds it and reacts with the allyl. A second channel ( $\pi-\eta^{3}$ pathway) is characterized by a $\pi-\eta^{3}$ interaction between the allyl fragment and the palladium, to which the $\mathrm{CO}$ molecule binds, before the two units react affording the product. In both cases, two consecutive migrations of the chlorine "assist" the course of the reaction. In a third case ( $\eta^{2}$ pathway) the allyl fragment initially enters the palladium coordination sphere, then the $\mathrm{CO}$ molecule simultaneously binds it and the phosphorous atom of one phosphine ligand. The first two paths are favoured.
\end{abstract}

Keywords: carbonylation, DFT, palladium, reaction mechanism, catalysis

\section{Introduction}

Mixtures of $\mathrm{Pd}(\mathrm{II})$ compounds, especially $\mathrm{Pd}(\mathrm{OAc})_{2}$ and tertiary phosphines are widely used in many catalytic reactions. Well known examples of this usage are Suzuki [1], Heck [2], Tsuji-Trost [3] and cross-coupling [4] reactions. In spite of the synthetic importance of these processes and the large amount of experimental and theoretical work available in literature, several issues still remain unclear. One 
certainly concerns the palladium oxidation state of the active catalytic complexes. Experimental and theoretical works either proposing $\mathrm{Pd}(\mathrm{II})$ complexes as active catalytic species or supporting the opposite idea (i.e. $\operatorname{Pd}(0)$ intermediates as true catalysts) can be found. Even if several experimental evidences support the hypothesis of a $\mathrm{Pd}(\mathrm{II})$ catalytic species [5-10], it is quite likely that both species can act as a catalyst [5-8], depending on the reaction conditions and the nature of the pre-catalyst complex. This hypothesis is enforced by the fact that $\mathrm{Pd}$ complexes can easily undergo redox transformations in both directions. Another interesting point, which certainly does not contribute to clarify the problem, is that an excess of phosphines enhances the catalytic rate. This promoting effect is assumed to be caused by the stabilization of the catalytic species. Since phosphines can form complexes either with $\operatorname{Pd}(0)$ or $\operatorname{Pd}(\mathrm{II})$, this evidence adds further uncertainty to the matter.

Furthermore, the mechanism of the possible conversion between the two oxidation states is not obvious. Kinetic studies carried out by Amatore [11] and coworkers for $\mathrm{Pd}(\mathrm{OAc})_{2} / \mathrm{PPh}_{3}$ mixtures show that the rate-limiting step is an intramolecular process that affords a $\operatorname{Pd}(0)$ species, which is unstable in the absence of a ligand excess. This species is characterized by two different ${ }^{13} \mathrm{P}$ NMR signals and reacts with iodobenzene. These authors have carried out additive oxidation of iodobenzene [12] to $\operatorname{Pd}(0)$ complexes together with cyclic voltammetry experiments (that allow to ascertain the amount of $\operatorname{Pd}(0)$ species) to establish the oxidation state (i.e. $\operatorname{Pd}(0))$ of the intermediates. When the reaction takes place in an excess of phosphines, it is possible to stabilize the $\mathrm{Pd}(0)$ species (that shows only one broad ${ }^{13} \mathrm{P}$ NMR signal). The authors propose the formation of anionic $\operatorname{Pd}(0)$ catalytic active species $\left[\mathrm{Pd}\left(\mathrm{PPh}_{3}\right)_{n}(\mathrm{OAc})_{2}\right]^{2-}(\mathrm{n}=2,3)$ and also suggest the following reaction mechanism for the reduction of $\mathrm{Pd}(\mathrm{OAc})_{2}$ by $\mathrm{PPh}_{3}$ : 
$\mathrm{Pd}(\mathrm{OAc})_{2}+2 \mathrm{PPh}_{3} \rightarrow \mathrm{Pd}(\mathrm{OAc})_{2}\left(\mathrm{PPh}_{3}\right)_{2}$ fast

$\mathrm{Pd}(\mathrm{OAc})_{2}\left(\mathrm{PPh}_{3}\right)_{2} \rightarrow \mathrm{Pd}^{0}\left(\mathrm{PPh}_{3}\right)(\mathrm{OAc})^{-}+\mathrm{AcO}-\mathrm{PPh}_{3}{ }^{+}$slow

$\mathrm{Pd}^{0}\left(\mathrm{PPh}_{3}\right)(\mathrm{OAc})^{-}+\mathrm{PPh}_{3} \rightarrow \mathrm{Pd}^{0}\left(\mathrm{PPh}_{3}\right)_{2}(\mathrm{OAc})^{-}$fast

$\mathrm{Pd}^{0}\left(\mathrm{PPh}_{3}\right)_{2}(\mathrm{OAc})^{-}+\mathrm{PPh}_{3} \rightarrow \mathrm{Pd}^{0}\left(\mathrm{PPh}_{3}\right)_{3}(\mathrm{OAc})^{-}$fast

$\mathrm{OAc}_{-} \mathrm{PPh}_{3}{ }^{+}+\mathrm{H}_{2} \mathrm{O} \rightarrow \mathrm{AcOH}+\mathrm{O}=\mathrm{PPh}_{3}+\mathrm{H}^{+}$fast

The existence and stability of $\operatorname{Pd}(0)$ anionic intermediates, i.e. $\mathrm{Pd}^{0}\left(\mathrm{PPh}_{3}\right)_{\mathrm{n}}(\mathrm{OAc})^{-}(\mathrm{n}=2,3)$ has been demonstrated either experimentally [14] or by means of theoretical calculations [15]. The role of water molecules in the reduction process has been investigated by Ozawa and collaborators [16]. They found that water molecules are necessary to produce $\mathrm{Pd}(0)$ species in the reaction of $\mathrm{Pd}(\mathrm{AcO})_{2}$ with three equivalents of BINAP. In addition, labelling experiments using ${ }^{18} \mathrm{O}$ suggest that the oxygen atom of the phosphine oxide comes from the acetate ligands.

The first purpose of the present paper is to clarify some of the abovementioned aspects. In particular we intend to shed light on the real nature of $\operatorname{Pd}(0)$ and Pd(II) complexes which form when Pd(II) compounds bearing oxygenated ligands (such as $\left.\mathrm{Pd}(\mathrm{OAc})_{2}\right)$ and tertiary phosphines are combined, as occurring in many reactions catalyzed by this metal. Furthermore we aim to understand how these complexes form from the starting reagents.

In addition to these aspects, the mechanism of carbonylation reactions of allyl halides catalyzed by $\operatorname{Pd}(0)$ complexes is investigated. We have chosen this reaction because of its importance in both academic and industrial environment: carbonyl group certainly represents one of the most versatile functional groups in organic synthesis. Transition metal-based carbonylation chemistry has been central to an extraordinary progress in catalysis during the last half century after the pioneering work by Roelen [17] on ethene hydroformylation catalyzed by cobalt tetracarbonyl 
hydride $\mathrm{HCo}(\mathrm{CO})_{4}$. The well-known Reppe carbonylation, for instance, is the result of an extensive research program in carbonylation chemistry carried out by Reppe and co-workers [18]. However, in all cases, high temperature (in the range $50^{\circ}-150^{\circ}$ ), high $\mathrm{CO}$ pressure, expensive autoclave equipment and large quantities of dangerous volatile and unstable catalysts such as $\mathrm{HCo}(\mathrm{CO})_{4}$ and $\mathrm{Fe}(\mathrm{CO})_{5}$ are required.

The severe conditions needed in Roelen and Reppe carbonylation processes remained approximately the same for more than twenty years. Nevertheless, during the last three decades, the work of many researchers, in particular Wilkinson, Heck and Tsuji [19], led to the development of new stable and effective catalysts based on rhodium and palladium. As a result, new carbonylation procedures under mild conditions (low temperature and pressure) are now available. To carry out many of these reactions, very small amounts of stable catalyst precursors such as $\mathrm{Pd}\left(\mathrm{PH}_{3}\right) \mathrm{Cl}_{2}$ and $\mathrm{RhCl}(\mathrm{CO})_{4}\left(\mathrm{PPh}_{3}\right)_{2}$ are necessary [20]. A special case is represented by the carbonylation of allyl halides. For this reaction several papers proposing different transition metal complexes as catalysts, have appeared [21-36]. Nickel tetracarbonyl $\mathrm{Ni}(\mathrm{CO})_{4}[22,31,37]$ was proposed for the first time by Chiusoli [22,31]. However, even if this reaction can be carried out at room temperature and under atmospheric or low CO pressures (2-3 atm.) [33], a large amount of nickel tetra-carbonyl is required. Since the direct manipulation of $\mathrm{Ni}(\mathrm{CO})_{4}$ is not easy because of its high toxicity, this species has been extensively replaced by palladium complexes. As a consequence, during the last three decades, several different reaction conditions and techniques have been proposed to improve the palladium-catalyzed carbonylation of allylic systems [38-50, 71-73].

Recently we have reported a theoretical investigation of the mechanism of palladium-catalyzed carbonylation of allylic systems to afford $\beta, \gamma$-unsaturated acyl 
derivatives [51-53]. A model-system consisting of the $\mathrm{Pd}\left(\mathrm{PH}_{3}\right) \mathrm{Cl}_{2}$ complex (emulating the real catalyst $\mathrm{Pd}\left(\mathrm{PPh}_{3}\right)_{2} \mathrm{Cl}_{2}$ with the metal in its +2 oxidation state), a CO molecule and allyl choride was used [53]. This study demonstrated the existence of two different reaction pathways leading to the $\beta, \gamma$-unsaturated acyl chloride. In one case ( $\eta^{2}$ pathway) the carbonylation proceeds via the formation of $\alpha$-allyl palladium intermediates. In the other case the process involves a direct attack of the $\mathrm{CO}$ molecule onto the metal ( $\sigma$ pathway). Along the $\eta^{2}$ pathway the process is characterized by a rather complex mechanism: the $\mathrm{CO}$ unit does not insert directly into a Pd-C bond, but interacts simultaneously with one of the two $\mathrm{Cl}$ ligands and the closest carbon atom of the allyl double-bond. This affords a cyclic structure including the acyl chloride group. The highest transition state along this path is $43.9 \mathrm{kcal} \mathrm{mol}^{-1}$ above reactants. A lower transition state $\left(33.4 \mathrm{kcal} \mathrm{mol}^{-1}\right.$ above reactants) characterizes the $\sigma$ pathway: this reaction channel is definitely favoured with respect to that involving $\eta^{2}$-complexes.

In the present study we examine in details the reaction mechanism of allyl chloride carbonylation catalyzed by $\operatorname{Pd}(0)$ species that form according to the mechanism above reported. This study should outline in details the mechanistic changes with respect to the $\mathrm{Pd}(\mathrm{II})$-based carbonylation of a similar system and permits the understanding of the effect of the oxidation state change [53]. $\mathrm{Pd}(0)$ species are considered in many cases to be the active catalytic species [13, 63-70], so a systematic study comparing both oxidation states will be very insightful. It is worth to mention that here a more realistic model-system including triphenylphosphine ligands instead of simple $\mathrm{PH}_{3}$ units (as done in previous papers) is considered. This should provide much more reliable information on the mechanism of this reaction. 


\section{Computational Procedures}

All DFT computations have been performed with the Gaussian 03 [54] series of programs using the non-local hybrid Becke's three-parameter exchange functional [55] denoted as B3LYP in the Gaussian formalism and a locally dense basis set (LDBS) approach [56]. According to the LDBS approach, the system has been partitioned into two different regions, which were assigned basis sets of different accuracy. One region contains the atoms which play the most important role in the reaction: the metal, the allyl moiety, the acetate fragments, the $\mathrm{CO}$ molecule and the phosphorous atoms. For the atoms belonging to this region we have used the DZVP basis, which is a Local Spin Density (LSD)-optimized basis set of double-zeta quality [57]. For the other region, comprised of all the remaining atoms (the phenyl rings), the $3-21 \mathrm{G}^{*}$ basis set [54] has been employed. When not otherwise specified, the reported energy values and/or geometrical parameters have been obtained with this basis denoted as DZVP/3-21G*.

To check the reliability of this approach we have carried out additional computations (with full geometry optimization) using the DZVP basis set on all atoms and we have compared the results to those obtained at the DZVP/3-21G* level. In these computations we have considered some of the key critical points located on the PES. We have found that the more accurate representation provides results (energetics and geometrical parameters) very similar to the DZVP/3-21G* basis. Thus, we can state with confidence that the $3-21 \mathrm{G}^{*}$ basis satisfactorily emulates the DZVP representation. These data are reported in details in the Supporting Information material.

We have performed frequency computations to determine the nature of the various critical points. In most cases, the shape of the transition vector provided by the frequency computations clearly indicates which intermediates a given transition state is connected to. However a series of IRC computations has been carried out starting from the most important transition states. The relative graphics and figures are available in the Supporting Information section. Furthermore, to roughly evaluate the solvent effects, single-point calculations have been carried out on the gas-phase optimized structures with the Polarized Continuous Model method (PCM based on the integral equation formalism model) [58] available in Gaussian 03: a dielectric 


\section{Results and Discussion}

\section{A. Palladium Oxidation-Reduction in Phosphine-Palladium Complexes: a Mechanistic Hypothesis.}

We have investigated the conversion and reduction mechanism of $\mathrm{Pd}(\mathrm{OAc})_{2}$ to $\mathrm{Pd}(0)$ complexes. The model-system consists of one $\mathrm{Pd}(\mathrm{OAc})_{2}$ molecule (palladium in the +2 oxidation state) and five tri-phenylphosphine $\left(\mathrm{PPh}_{3}\right)$ molecules which come into play at different stages of the process. All energy values discussed in this section are relative to these non-interacting species, which represent the asymptotic limit (AL).

The consecutive addition of two triphenylphosphine units to the starting $\mathrm{Pd}(\mathrm{OAc})_{2}$ complex occurs in the first stage of the reaction. Although $\mathrm{Pd}(\mathrm{OAc})_{2}$ in its crystal structure is actually a trimer, we believe that the monomer is a good model for the calculations, since we can expect that the monomeric complex $\operatorname{Pd}(\mathrm{OAc})_{2}\left(\mathrm{PPh}_{3}\right)_{2}$ will be formed via an analogous mechanism from the trimer. The corresponding energy profile is shown in Figure 1. A schematic representation of the structure of the various critical points is given in Scheme 1 and selected bond lengths are collected in Table 1. It is interesting to outline that, each time a new phosphine ligand is added to the system, shallow minima characterized by weak interactions precede the actual 
formation of phosphorous-metal bonds. These interactions (mainly H-bonds between the palladium ligands and the incoming phenyl rings) significantly weaken in a solvent phase because of electrostatic effects (the PCM values are reported in round parenthesis). For completeness, all these points will be discussed. Another important structural feature of the species that form upon addition of $\mathrm{PPh}_{3}$ molecules, is the well-known palladium propensity to reach a tetra-planar coordination. When the four ligands are not remarkably cumbersome and this configuration becomes possible, a strong energy gain is observed.

In the starting $\mathrm{Pd}(\mathrm{OAc})_{2}$ complex (M0) the two acetate fragments chelate the metal and saturate the four coordination sites of palladium, thus leading to an approximately square planar structure. The preliminary complex M1 forms without overcoming any energy barrier upon addition of one phosphine ligand with a stabilization $\Delta \mathrm{E}$ of $4.7 \mathrm{kcal} \mathrm{mol}^{-1}$. Weak interactions involving one aromatic ring, the metal and one acetate ligand can be detected in M1. As mentioned above, this stabilization is significantly reduced when solvent effects are considered $(\Delta \mathrm{E}=-0.6$ kcal mol${ }^{-1}$ ). A further approach of $\mathrm{PPh}_{3}$ to the metal involves a transition state (TS1, energy barrier of $0.9 \mathrm{kcal} \mathrm{mol}^{-1}$ ), which remains lower than M0. Thus, the binding of $\mathrm{PPh}_{3}$ to palladium is a rather easy process that causes the almost complete breaking of one Pd-O bond, as observed in the resulting intermediate M2. The new Pd-P1 bond ( $2.304 \AA$ ) is determined by the interaction between the phosphorus lone pair and the empty metal d orbitals. It replaces the weaker Pd-O1 bond $(\mathrm{Pd}-\mathrm{O} 1=3.008 \AA)$ and results in a significant stabilization of the new complex $\mathbf{M} 2$, which is $29.5 \mathrm{kcal} \mathrm{mol}^{-1}$ lower than M0 (28.3 kcal mol $\mathrm{k}^{-1}$ in the presence of solvent effects). A further contribution to the overall stabilization is probably due to a strain decrease of the palladium-acetate oxygen structure and the corresponding strengthening of the $\mathrm{Pd}-\mathrm{O} 2$ 
bond. In particular, the replacement of one oxygen with a phosphorous atom allows a more regular and stable square planar coordination of the metal.

(Table 1 here)

(Figure 1 here)

The energy profile for the addition of a second phosphine ligand is almost identical to that previously discussed. A slight stabilization accompanies the formation of a new preliminary complex $\mathbf{M 3}\left(4.3 \mathrm{kcal} \mathrm{mol}^{-1}\right.$ lower than $\mathbf{M 2}$ and 33.8 kcal $\mathrm{mol}^{-1}$ lower than M0). The weak interactions that stabilize this intermediate (again weak H-bonds) are totally lost in condensed phase, where $\mathbf{M 3}$ is $26.7 \mathrm{kcal} \mathrm{mol}^{-}$ ${ }^{1}$ below M0 and hence $1.6 \mathrm{kcal} \mathrm{mol}^{-1}$ higher than M2. Even if this marked destabilization of M3 is partly due to the frozen geometry (only PCM single point calculations have been carried out), these results suggest that the intermediate M3 does not probably exist in condensed phase or that it is in equilibrium with $\mathbf{M 2}$.

(Scheme 1 here)

The definitive binding of the second phosphine to palladium is a crucial point of the process. As a matter of fact, the resulting complex $\mathrm{Pd}(\mathrm{OAc})_{2}\left(\mathrm{PPh}_{3}\right)_{2}$ can exist in two different configurations (cis and trans) and each of them originates a unique reaction pathway (denoted here as cis and trans channel) leading to palladium reduction.

We first discuss the trans-channel. The corresponding energy profile is depicted in Figure 2. The various structures located along the reaction path are 
schematically represented in Scheme 2 and 3, and the most important geometrical parameters are reported in Table 2 and 3. From M3, the transition state TS2 leads to the formation of the trans complex M4. In TS2 the length of the new forming phosphorus-palladium bond (P2-Pd) is $3.040 \AA$ (i.e. almost identical to the value found in TS1) whereas the breaking oxygen-palladium bond $\mathrm{Pd}-\mathrm{O} 3$ is still rather short: $2.377 \AA$. The corresponding activation barrier $(\mathrm{E}(\mathbf{T S 2})-\mathrm{E}(\mathbf{M 3}))$ is $4.5 \mathrm{kcal}$ $\mathrm{mol}^{-1}$ and does not change significantly in the presence of solvent effects $(4.1 \mathrm{kcal}$ $\mathrm{mol}^{-1}$ ). It is reasonable to believe that a steric hindrance around palladium makes this barrier higher than that associated to TS1. The trans complex M4 (Pd(OAc) $\left.)_{2}\left(\mathrm{PPh}_{3}\right)_{2}\right)$ exists in a typical planar tetra-coordinated configuration and is characterized by a strong energy stabilization: $17.3 \mathrm{kcal} \mathrm{mol}^{-1}$ with respect to $\mathbf{M} 3$ and $51.1 \mathrm{kcal} \mathrm{mol}^{-1}$ below the AL (this value becomes $45.2 \mathrm{kcal} \mathrm{mol}^{-1}$ in the presence of solvent). In M4, the oxidation state of palladium is still unchanged i.e. +2 .

(Table 2 here)

(Table 3 here)

(Figure 2 here)

A barrierless rotation around $\mathrm{Pd}-\mathrm{O} 2$ connects $\mathrm{M} 4$ to the intermediate M5 where the oxygen atom $\mathrm{O} 1$ is now facing the phosphorus atom $\mathrm{P} 1(\mathrm{O} 1-\mathrm{P} 1=2.884 \AA$ ). In spite of the new O1-P1 interaction, this structure is slightly higher in energy than M4 (2.5 and $3.4 \mathrm{kcal} \mathrm{mol}^{-1}$ in gas-phase and solvent, respectively). This is probably due to the unfavourable steric interactions among the phosphine phenyl rings, which must stay closer to accommodate the acetate fragment after rotation. The structural arrangement of M5 anticipates the next crucial step i.e. the palladium reduction that 
requires the transfer of the two acetate fragments to the phosphorus atom P1 (that becomes oxidized). This process occurs in two steps and involves a third phosphine, which enters the palladium coordination sphere. In the first step (transition state TS3) one oxygen atom of each acetate forms two almost complete bonds with the phosphorus atom $\mathrm{P} 1(\mathrm{P} 1-\mathrm{O} 1=1.861 \AA$ and $\mathrm{P} 1-\mathrm{O} 3=1.904 \AA$ ). Simultaneously the bond between the palladium and the second oxygen atom of each acetate significantly elongates. The lengths of these two palladium-oxygen bonds underline the asynchronous character of the acetate migration, Pd-O4 (2.438 $\mathrm{A})$ being shorter than Pd-O2 (2.539 $\AA$ ). A simultaneous removal of the phosphorus from the palladium is observed ( $\mathrm{P} 1-\mathrm{Pd}=2.478 \AA$ ). To balance the consequent loss of electron density on the metal orbitals, one of the phenyl group moves closer to the palladium, thus donating a certain amount of charge from the ring $\pi$ system (in particular, two carbon atoms of the benzene ring become rather close to the metal, the corresponding carbonpalladium distances being 2.402 and $2.686 \AA$ ). Thus, this transition state describes a situation where ligands and electron density are exchanged between the phosphorus (P1) and the palladium atoms, which are involved in a oxidation and reduction process, respectively. Also, the phosphorus atom (P1) is becoming penta-coordinated, because the low energy d-orbitals make possible the interaction with the oxygen atoms of the migrating acetate. Even though penta-coordinated phosphorus complexes may be quite unusual, they have been characterized experimentally [61]. TS3 is 25.5 $\mathrm{kcal} \mathrm{mol}^{-1}$ above the previous intermediate (M5) and $22.7 \mathrm{kcal} \mathrm{mol}^{-1}$ below the AL (in the presence of solvent these values become 33.9 and $7.9 \mathrm{kcal} \mathrm{mol}^{-1}$, respectively). TS3 leads to M6, which is $35.8 \mathrm{kcal} \mathrm{mol}^{-1}$ below M0, in which both acetates have completed the migration to phosphorus, even if in one case a weak interaction with palladium is still observed $(\mathrm{Pd}-\mathrm{O} 2=3.743 \AA$ ). The $\pi$ system of one phenyl ring of the 
leaving phosphine strongly interacts with the metal. The aromatic ring is simultaneously bonded to P1 and behaves as a metal ligand, allowing a significant charge transfer to the palladium and determining a strong stabilization of the system, as it has been observed in this kind of processes [13].

(Scheme 2 here)

(Scheme 3 here)

The redox process is completed with the transition state TS4 (second step) that corresponds to the definitive detachment of the phosphorus atom (bearing five ligands) from palladium. The phosphorous ligands include now the original three phenyl rings (one is still partly bonded to Pd) and the two acetates fragments. The separation of the five-coordinated phosphorous is helped by the approach of a new phosphine ligand to the metal $(\mathrm{P} 3-\mathrm{Pd}=3.544 \AA$ ), which has now a unsaturated coordination sphere. The resulting intermediate $\mathbf{M} 7$ (41.8 $\mathrm{kcal} \mathrm{mol}^{-1}$ below the starting reactants in gas phase and $36.2 \mathrm{kcal} \mathrm{mol}^{-1}$ in solvent) is formed by the $\mathrm{Pd}\left(\mathrm{PPh}_{3}\right)_{2}$ and $\mathrm{PPh}_{3}(\mathrm{OAc})_{2}$ complexes. The two acetates that coordinate the palladium in the starting species have been replaced by two phosphines. Thus, the metal has been reduced and the resulting oxidation state is now zero, while the phosphorus oxidation state has increased to five. The diagram of Figure 2 shows that the final species M7 is higher in energy than M4. This is mainly a consequence of the "holes" in the coordination sphere of palladium which can accept two more phosphine ligands, thus restoring the optimum tetra-coordination state.

We must stress an additional aspect concerning the redox process. Since TS4 is almost degenerate to M6, it is more cautious to consider this transition state as a 
computational shortcoming (which could easily disappear when changing the computational level) and the redox transformation a very asynchronous concerted process occurring in two phases. Furthermore, it is important to note that the redox transformation appears to be the rate-determining step of the whole process. This would be in agreement with the results of experimental kinetic studies [11] for mixtures $\mathrm{Pd}(\mathrm{OAc})_{2} / \mathrm{PPh}_{3}$ that show that the rate-limiting step of the reaction is an intra-molecular endothermic process affording a $\operatorname{Pd}(0)$ species. On the other hand, it is important to mention that the presence of a third ligand does not imply that the reaction is first order in phosphine, since it remains always at a non bonding distance from the palladium atom, and it is not involved in the bond breaking and forming process (TS3). Indeed, the interaction between the metallic centre and such a $\mathrm{PPh}_{3}$ is very week and could be suppressed for instance if the solvent was considered explicitly, so the proposed mechanism is still zero order in $\mathrm{PPh}_{3}$, as it was determined experimentally.

The binding of two additional phosphines to $\mathrm{Pd}\left(\mathrm{PPh}_{3}\right)_{2}$ again involves the preliminary formation of weakly bound intermediates corresponding to shallow minima on the potential surface. One phosphine ligand weakly interacts with $\mathrm{Pd}\left(\mathrm{PPh}_{3}\right)_{2}$ and affords M8 without overcoming any activation barrier. M8 is $45.8 \mathrm{kcal}$ $\mathrm{mol}^{-1}$ below the asymptotic limit $\left(35.8 \mathrm{kcal} \mathrm{mol}^{-1}\right.$ at the PCM level). The energy barrier ( $\mathrm{E}($ TS5) - E(M8)) for the definitive phosphine binding leading to M9 is very low, being only $0.23 \mathrm{kcal} \mathrm{mol}^{-1}\left(0.92 \mathrm{kcal} \mathrm{mol}^{-1}\right.$ when solvent effects are included). M9 is a planar tri-coordinated palladium complex $\mathrm{Pd}\left(\mathrm{PPh}_{3}\right)_{3}$ which lies $55.6 \mathrm{kcal} \mathrm{mol}^{-}$ ${ }^{1}$ below reactants $\left(45.0 \mathrm{kcal} \mathrm{mol}^{-1}\right.$ in the presence of solvent). The addition of a further phosphine follows the same pattern. M10 is the preliminary weakly bound complex, (60.1 kcal mol ${ }^{-1}$ more stable than reactants; $43.2 \mathrm{kcal} \mathrm{mol}^{-1}$ at the PCM level) and 
TS6 is the transition state leading to the formation of the final tetra-coordinated complex M11, which adopts a tetrahedral structure, typical of $\mathrm{d}^{10}$ metals like $\operatorname{Pd}(0)$ that also minimizes the repulsion between the cumbersome $\mathrm{PPh}_{3}$ ligands. This steric hindrance is responsible for the small stabilization associated with the entering of the forth phosphine in the metal coordination sphere: M11 lies $62.4 \mathrm{kcal} \mathrm{mol}^{-1}$ below the reactants, and only 2.3 lower than M10 (these values become 44.4 and $1.2 \mathrm{kcal} \mathrm{mol}^{-1}$, respectively, in the presence of solvent). Selected bond lengths for the intermediate complexes (M8, M9, M10 and M11) and the corresponding transition structures (TS5 and TS6) are collected in Table 4. In summary, the role of the additional phosphines is mostly to stabilize the resulting $\operatorname{Pd}(0)$ species. This result agrees this the fact that in the absence of one excess of ligand the products decompose rapidly.

(Table 4 here)

As above mentioned, a second reaction path (denoted as cis-channel) originates from M3. The energy profile is reported in Figure 2 and a schematic representation of the structures located on the initial part of this path is given in Scheme 4. The corresponding geometry parameters are collected in Table 5. A transition state $\mathbf{T S 7}$ leads from $\mathbf{M 3}$ to a $\left(\mathrm{Pd}(\mathrm{OAc})_{2}\left(\mathrm{PPh}_{3}\right)_{2}\right.$ cis complex M12, by overcoming an energy barrier $(\mathrm{E}(\mathbf{T S} 7)-\mathrm{E}(\mathbf{M 3}))$ of $10.3 \mathrm{kcal} \mathrm{mol}^{-1}\left(7.4 \mathrm{kcal} \mathrm{mol}^{-1}\right.$ in solvent). This barrier is $5.8 \mathrm{kcal} \mathrm{mol}^{-1}$ larger than that associated with transition state TS2 leading to the trans complex and M12 is $7.0 \mathrm{kcal} \mathrm{mol}^{-1}$ higher in energy than the corresponding trans-complex M4. This difference is certainly caused by the steric repulsion between the two adjacent phosphine ligands. The key step for Pd oxidation, i.e. transfer of the two acetate ligands from the metal to the phosphine phosphorous atom, involves the transition state TS8 where the migration of the two acetate 
fragments follows a mechanism slightly different with respect to that previously discussed for the trans complex. An additional, third phosphine ligand (not bound to palladium) is immediately involved, entering the system at this stage. The corresponding phosphorus atom P3 interacts with the two acetates. In particular the oxygen $(\mathrm{O} 2)$ of one acetate is bridging the $\mathrm{Pd}$ and $\mathrm{P} 3$ atoms: the $\mathrm{P} 3-\mathrm{O} 2$ interaction is already rather strong $(2.130 \AA)$ and the $\mathrm{Pd}-\mathrm{O} 2$ bond remains significant $(\mathrm{Pd}-\mathrm{O} 2=$ $2.649 \AA$ ). Even the second oxygen of the same acetate (O1) considerably interacts with the phosphorus $(\mathrm{P} 3-\mathrm{O} 1=2.572 \AA)$. The second acetate fragment remains closer to the metal $(\mathrm{Pd}-\mathrm{O} 3)=2.252 \AA)$ and its interaction with the approaching phosphine is much weaker $(\mathrm{P} 3-\mathrm{O} 2=2.941 \AA)$. In summary, the incoming phosphine "captures" the two acetates helping their expulsion from the coordination sphere of the metal that retains the electrons of the Pd-OAc bonds, thus reducing its oxidation state. However, TS8 is characterized by a rather high energy barrier $\left(41.8 \mathrm{kcal} \mathrm{mol}^{-1}\right.$ in gas phase and $52.2 \mathrm{kcal} \mathrm{mol}^{-1}$ in solvent) and lies $20.3 \mathrm{kcal} \mathrm{mol}^{-1}\left(19.7 \mathrm{kcal} \mathrm{mol}^{-1}\right.$ at the PCM level) above the highest transition state located along the trans pathway. Thus, the cis channel cannot effectively compete with the trans one and for this reason we do not discuss further.

(Table 5 here)

(Scheme 4 here)

(Figure 3 here)

The phosphorous species that forms after TS4 $\left(\mathrm{PPh}_{3}(\mathrm{OAc})_{2}\right)$ easily converts into the corresponding phosphine oxide, which is detected experimentally at the end of the reaction, by reaction with one water molecule. The intermediates and transition states along this path are represented in Scheme 5, while the energy profile is 
displayed in Figure 3. From now on, we refer to the separated reagents of this process $\left(\mathrm{PPh}_{3}(\mathrm{OAc})_{2}\right.$ and one water molecule $)$ as M13. The interaction between these two species leads to the barrierless formation of a complex (M14) characterized by a hydrogen bond between $\mathrm{H} 1$ and $\mathrm{O} 2$ that keeps the two molecules together and lowers the energy of the system by $7.4 \mathrm{kcal} \mathrm{mol}^{-1}$ with respect to the isolated reactants. As expected this energy stabilization is smaller when the solvent effects are introduced in a PCM calculation and becomes $2.9 \mathrm{kcal} \mathrm{mol}^{-1}$. In the next step (TS9), the water molecule closely approaches the phosphorus atom causing a significant series of chemical transformations, which directly lead to the product. In TS9, the water molecule is strongly interacting with the phosphorus: the O5-P1 distance is $1.918 \AA$. Simultaneously, each of the two water hydrogen atoms is hopping to one acetate oxygen, namely the one which is not bonded to the phosphorus atom. This double transfer is not synchronous: $\mathrm{H} 1$ is almost equally shared between $\mathrm{O} 2$ and $\mathrm{O} 5$ (H1-O2 $=1.172 \AA$ and $\mathrm{H} 1-\mathrm{O} 5=1.244 \AA$ ), while $\mathrm{H} 2$ is only weakly interacting with $\mathrm{O} 4$ (H2$\mathrm{O} 4=1.968 \AA$ ). The behaviour of the two acetates on the phosphorus is analogous: the P1-O1 bond has evidently elongated $(\mathrm{P} 1-\mathrm{O} 1=2.131 \AA)$ so that an acetic acid molecule is almost formed and being expelled from the phosphorus coordination shell. On the contrary, the corresponding P1-O3 bond, because of the weakening of the trans effect associated to Pd-O1, is shorter than in M14 (1.750 $)$ : the acetate is still bonded to the phosphorus, which is octahedrally coordinated. The TS9 transition state lies $9.3 \mathrm{kcal} \mathrm{mol}^{-1}$ above the isolated reactants $\left(\mathrm{PPh}_{3}(\mathrm{OAc})_{2}+\mathrm{H}_{2} \mathrm{O}\right)$ and the relative activation energy with respect to the previous intermediate (M14) is $16.7 \mathrm{kcal}$ $\mathrm{mol}^{-1}$. The energy barrier rises to $21.5 \mathrm{kcal} \mathrm{mol}^{-1}$ at the PCM level, because the stabilizing hydrogen bond network loses strength and importance when the solvent effects are considered. On the other hand, since the precursor M7 lies already 41.8 
$\mathrm{kcal} \mathrm{mol}^{-1}$ below the asymptotic limit $\left(36.2 \mathrm{kcal} \mathrm{mol}^{-1}\right.$ when the solvent is considered), it is reasonable to assume that the system will have energy enough to overcome the barrier easily. In spite of the above-mentioned asynchronicity, an IRC calculation demonstrated that TS9 is directly connected to the final product of this process: M15. This species corresponds to the phosphine oxide $\mathrm{Ph}_{3} \mathrm{P}=\mathrm{O}$ (where the phosphorous has still the formal oxidation state +5 ) interacting with two acetic acid molecules. The phosphine oxide features a tetrahedral geometry and the two hydrogen bonds which keep this complex close to the acetic acid molecules are almost identical: H1-O5 = $1.728 \AA$ and $\mathrm{H} 2-\mathrm{O} 5=1.735 \AA$. M15 lies largely below the initial reactants: $47.1 \mathrm{kcal}$ $\mathrm{mol}^{-1}$ (37.8 $\mathrm{kcal} \mathrm{mol}^{-1}$ at the PCM level). In conclusion, the formation of the phosphine oxide is strongly exothermic and characterized by an energy barrier quite small: hence the process seems to be rather easy.

(Table 6 here)

(Scheme 5 here)

\section{B. Allyl Chloride Carbonylation Catalyzed by $\mathrm{Pd}(0)$ Complexes}

In this section, we discuss the carbonylation reaction of allyl chloride catalyzed by $\operatorname{Pd}(0)$ complexes that easily form when $\mathrm{Pd}(\mathrm{OAc})_{2}$ species are used in the presence of an excess of phosphines. To this purpose we use a model-system consisting of $\mathrm{Pd}\left(\mathrm{PPh}_{3}\right)_{4}$, a $\mathrm{CO}$ molecule and the allyl chloride molecule. These three noninteracting chemical species represent now the asymptotic limit (AL).

(Figure 4 here) 


\section{B.1. The $\sigma$-Pathway.}

This pathway ( $\sigma$-pathway) originates from a direct, weak and initial interaction of the chlorine atom with the metal and leads to the formation of allyl-palladium $\sigma$ complexes. The corresponding reaction profile is shown in Figure 4 and a schematic representation of the various structures located along it is given in Scheme 6. Selected bond lengths for the critical points are reported in Table 7. Palladium complexes such as $\mathrm{Pd}\left(\mathrm{PPh}_{3}\right)_{4}$ are characterized by a high steric hindrance, which makes difficult the entering of an additional group in the metal coordination sphere. To avoid the formation of penta-coordinated transient species (bipyramidal configuration) with strong steric repulsions, the replacement of one ligand by a new group must occur in two steps. Thus, in the present case, the expulsion of one phosphine from $\operatorname{Pd}\left(\mathrm{PPh}_{3}\right)_{4}$ anticipates the binding of a chloroallyl molecule, as a new ligand. This stage $\left(\right.$ expulsion of $\mathrm{PPh}_{3}$ ) is the same as the reverse of the final step (M9 $\rightarrow$ M11) of the process described in the previous section and will not be discussed again. In the resulting trigonal complex $\mathrm{Pd}\left(\mathrm{PPh}_{3}\right)_{3}$ there is now enough room to allow the allyl unit to approach the metal. This affords a preliminary intermediate, M16, where a very weak interaction involving the chlorine atom $(\mathrm{Cl}-\mathrm{Pd}$ distance $=4.697 \AA)$ binds the allyl molecule to the metal. In M16, which is $3.0 \mathrm{kcal} \mathrm{mol}^{-1}$ above the AL (1.6 kcal $\mathrm{mol}^{-1}$ below when solvent effects are included), the metal retains its planar trigonal configuration. When a $\mathrm{CO}$ molecule approaches the complex, a more stable complex (M17 $8.1 \mathrm{kcal} \mathrm{mol}^{-1}$ below the AL) forms, through a shallow transition state TS10: the new bond $(\mathrm{Pd}-\mathrm{C} 4=2.015 \AA)$ is quite strong despite the crowded coordination sphere. Actually the important step of the reaction is the next one when the CO molecule establishes a simultaneous interaction with the palladium and the allyl group (transition state TS11). In TS11 the carbon atom C4 of the CO unit forms two new bonds: one with palladium and one with the $\mathrm{C} 1$ allyl carbon. The formation of the $\mathrm{C} 1$ - 
C4 bond (1.912 $\AA$ ) causes important structural changes in the allyl moiety: while the C2-C3 bond increases its double bond character (C2-C3 = 1.399 $\mathrm{A}), \mathrm{C} 1-\mathrm{C} 2$ is turning into a single bond $(\mathrm{C} 1-\mathrm{C} 2=1.419 \AA)$. As a consequence the chlorine atom migrates from C3 (allyl system) to palladium: the $\mathrm{C} 3-\mathrm{Cl}$ and $\mathrm{Pd}-\mathrm{Cl}$ bond lengths are 2.392 and $4.654 \AA$, respectively. This structural rearrangement also leads to a significant weakening of the Pd-P3 bond (2.626 ̊): for electronic and, above all, steric reasons, the palladium cannot bear so many ligands. The remarkable migrating and bridging attitude of chlorine and halogens in general has already been observed in several systems of this kind and it has been demonstrated to represent a crucial step in the carbonylation reaction $[62,52,53]$. An approximate tetrahedral structure of palladium characterizes TS11. Once again, the presence of five groups and the steric hindrance of the cumbersome phenyl rings prevents palladium to adopt the more typical planar tetra-coordination. This transition state is $24.9 \mathrm{kcal} \mathrm{mol}^{-1}$ above the asymptotic limit $\left(15.0 \mathrm{kcal} \mathrm{mol}^{-1}\right.$ at the PCM level) and the corresponding barrier (E(TS11) - E(M17) is $33.0 \mathrm{kcal} \mathrm{mol}^{-1}\left(26.8 \mathrm{kcal} \mathrm{mol}^{-1}\right.$ in solvent). It is important to point out that in this step, which is rate-determining for this pathway, the Pd formal oxidation state is changing from 0 to +2 .

(Scheme 6 here)

(Table 7 here)

The resulting intermediate (M18) is rather stable being $13.2 \mathrm{kcal} \mathrm{mol}^{-1}$ below the asymptotic limit. The palladium has reached a square planar tetra-coordination and is surrounded by one chlorine atom, the ally carbonyl chain and two phosphines. The third phosphine has been almost definitely expelled (the Pd-P3 bond length is $4.291 \AA$ ) and the Pd oxidation state is now unquestionably +2 . In the subsequent step 
(transition state TS12), we observe a back migration of the chlorine atom from the palladium to the carbonyl atom $\mathrm{C} 4$; the Pd-carbon bond breaks ( $\mathrm{Pd}-\mathrm{C} 4=2.384 \AA$ ) and a phosphine enters again the palladium coordination sphere $(\mathrm{Pd}-\mathrm{P} 3$ bond length $=$ $2.698 \AA$ A). Hence, the palladium oxidation state changes again: from +2 to 0 . It is interesting to highlight the role of the chlorine atom which "assists" the carbonylation process. Its double migration forward and backward the metal allows to complete the carbonyl insertion and to undergo the internal rearrangement of the allyl moiety necessary to afford the final product i.e. the acylchloride and the $\operatorname{Pd}\left(\mathrm{Ph}_{3}\right)_{3}$ complex (6.4 $\mathrm{kcal} \mathrm{mol}^{-1}$ below reactants). An additional phosphine ligand can approach the metal, give back the regenerated catalyst $\mathrm{Pd}\left(\mathrm{PPh}_{3}\right)_{4}$ (lying $13.4 \mathrm{kcal} \mathrm{mol}{ }^{-1}$ below the AL), thus closing the catalytic cycle.

\section{B.2 .The $\pi \eta^{3}$-allyl-palladium complexes pathway}

As previously shown, after the initial displacement of a phosphine, in $\mathrm{Pd}\left(\mathrm{PPh}_{3}\right)_{3}$, the metal can accept a chloro-allyl group as new ligand. However, this time, the resulting complex, M19 (see Scheme 7), features a $\pi-\eta^{2}$ metal coordination through the C1-C2 double bond that gives rise to an actual binding between $\mathrm{Cl}-\mathrm{CH}_{2}-\mathrm{CH}=\mathrm{CH}_{2}$ and the palladium. M19 is a tetrahedral intermediate where the typical donation/back donation pattern can be observed: electron density is donated from the $\mathrm{C} 1=\mathrm{C} 2 \pi$ bonding orbital to the empty metal d-orbitals and back-donated from the filled d-orbitals to the $\mathrm{C} 1=\mathrm{C} 2 \pi *$ anti-bonding orbital. The slight elongation of the $\mathrm{C}=\mathrm{C}$ bond $(\mathrm{C} 1-\mathrm{C} 2=1.373 \AA)$ is a clear indication of this situation. M19 is rather unstable and lies $7.9 \mathrm{kcal} \mathrm{mol}{ }^{-1}$ above reactants: $\left(\mathrm{Pd}\left(\mathrm{PPh}_{3}\right)_{4}+\right.$ chloro-allyl $\left.+\mathrm{CO}\right)$. It is also higher in energy than $\mathrm{Pd}\left(\mathrm{PPh}_{3}\right)_{3}+$ chloroallyl $+\mathrm{CO}+\mathrm{PPh}_{3}$ : therefore, the binding of the allyl fragment to $\mathrm{Pd}\left(\mathrm{PPh}_{3}\right)_{3}$ destabilizes the system, probably for steric reasons that 
prevent stronger interaction between the ligands and the metal. The incapability of the palladium to adopt the typical and preferred square-planar coordination reflects this quite unstable setting. As a matter of fact, the displacement of one phosphine from M19 can very easily take place through the next transition state, TS13, which is associated to an energy barrier of $0.2 \mathrm{kcal} \mathrm{mol}^{-1}$ only. This leads to a complex, M20, in which the palladium is coordinated by three ligands only and which is $3.3 \mathrm{kcal} \mathrm{mol}^{-}$ ${ }^{1}$ more stable than the three-phosphine complex M19 (and $4.6 \mathrm{kcal} \mathrm{mol}^{-1}$ above the AL). At the PCM level, this complex is, on the contrary, a little bit more stable than the AL: $1.5 \mathrm{kcal} \mathrm{mol}^{-1}$. As observed several times in the present study, the loss of a phosphine from crowed tetra-coordinated palladium complexes is easy and may lead to a negative energy variation as consequence of the strengthening of the remaining metal-ligands interactions. In M20, the C1-C2 double bond is a little longer (1.396 ̊̊) indicating that the allyl-palladium bond has become stronger. Also the two phosphines on the palladium move closer and strengthen their bond with the metal. Furthermore, a very loose interaction between the palladium and the expulsed phosphine can still be detected $(\mathrm{Pd}-\mathrm{P} 3=4.877 \AA$ ).

(Table 8)

In the next transition state (TS14), the chlorine atom shows once again its great migrating attitude, passing from the allyl group (namely the $\mathrm{C} 3$ carbon atom) to the palladium. Also in this case, it is interesting to highlight the long distance between the halogen atom and the metal $(\mathrm{Cl}-\mathrm{C} 3=3.298 \AA)$ : it is evident that these two centres do not need to closely approach each other for their interaction to be effective. The analysis of the eigenvector associated to the negative eigenvalue of the Hessian matrix indicates that also the approaching of $\mathrm{C} 3$ to the palladium is actually taking place. 
TS14 is located $22.2 \mathrm{kcal} \mathrm{mol}^{-1}$ above the reactants $\left(\mathrm{Pd}\left(\mathrm{PPh}_{3}\right)_{4}+\right.$ chloroallyl $\left.+\mathrm{CO}\right)$ and its intrinsic activation energy $(\mathrm{E}(\mathbf{T S 1 4})-\mathrm{E}(\mathbf{M 2 0}))$ is $17.6 \mathrm{kcal} \mathrm{mol}^{-1}(12.5$ at the PCM level). The Pd formal oxidation state is passing from 0 to +2 . It is important to underline that this critical point, that results to be the highest along the reaction channel that is being discussed, is slightly lower in energy than TS11, the highest transition state located on the $\sigma$-pathway described in the previous section: by $2.7 \mathrm{kcal}$ $\mathrm{mol}^{-1}\left(2.8 \mathrm{kcal} \mathrm{mol}^{-1}\right.$ at the PCM level). Another significant result is that an analogous transition state describing the migration of the chlorine atom, in which three (and not two) phosphines are bonded to the palladium (i.e. proceeding directly from M19) lies $5.4 \mathrm{kcal} \mathrm{mol}^{-1}$ above TS14, so that the pathway featuring two phosphines on the metal is favoured. As above-anticipated, the migration of the chlorine onto the palladium has the consequence of yielding a $\pi \eta^{3}$ type interaction between the allyl fragment and the metal centre (M21) in which all the three carbon atoms are practically at the same distance from the palladium: $\mathrm{C} 1-\mathrm{Pd}=2.247 \AA$ A, $\mathrm{C} 2-\mathrm{Pd}=2.251 \AA$ and $\mathrm{C} 3-\mathrm{Pd}=$ $2.268 \AA$. In this configuration, being still bonded to two phosphines, the palladium electronic shell is saturated (just like it was surrounded by the usual four ligands). For this reason, the chlorine atom remains partially detached from the metal (as noted above, chlorine-palladium interactions can be effectual even at large distances): the Cl-Pd distance is $2.968 \AA$, about $0.5 \AA$ longer than an ordinary chlorine-palladium bond. Also the slight separation of the third "spectator" phosphine that is now basically not interacting with the metal (Pd-P3 = 5.124 $\AA$ ) has certainly the same motivation. All these transformations do not lead to any significant energy variation though: M21 has the same energy as M20. The subsequent step (TS15) is, once more, an easy removal of a phosphine that allows to make room around the palladium coordination sphere for the incoming new ligand. Even in this case, the process is 
favoured by the strengthening of the interaction between the palladium and the groups, which remain bonded to it. As a matter of fact in TS15, while one phosphine moves away $(\mathrm{Pd}-\mathrm{P} 2=2.905 \AA)$, the allyl moiety and especially the chlorine atom (which stayed farther in M21) approach the metal ( $\mathrm{Pd}-\mathrm{Cl}=2.576 \AA$ ). In this case, the energy activation is higher than for TS13: $5.8 \mathrm{kcal} \mathrm{mol}^{-1}\left(7.7 \mathrm{kcal} \mathrm{mol}^{-1}\right.$ at the PCM level) because, in this case, not only the breaking of the palladium-phosphorous is taking place, but also a motion of the chlorine atom, which moves to a trans position with respect to the allyl fragment. Hence in the next complex, M22, the palladium is surrounded by a chlorine atom, the ally fragment (again in a $\pi-\eta^{3}$ fashion) and a single phosphine. The expulsed phosphine still retains a partial, weak interaction with the metal ( $\mathrm{P} 2-\mathrm{Pd}=4.540 \AA$ ), while, as anticipated above, the chlorine is now $0.53 \AA$ closer to it with respect to $\mathbf{M} 21(\mathrm{Pd}-\mathrm{Cl}=2.440 \AA)$. Also in this case, the loss of a phosphine causes only a small energy change, $\mathbf{M 2 2}$ being $0.8 \mathrm{kcal} \mathrm{mol}^{-1}$ above M21 and $5.4 \mathrm{kcal} \mathrm{mol}^{-1}$ above the reactants.

(Table 9 here)

The next stage involves the entering of the $\mathrm{CO}$ molecule into the palladium coordination sphere (TS16). The approach of the new ligand, which is forming the new C4-Pd bond (2.244 $\AA$ ), causes a change of the way through which the allyl unit interacts with the metal: one carbon atom moves away from the palladium $(\mathrm{Pd}-\mathrm{C} 1=$ $2.253 \AA$ ), while another metal-carbon bond (Pd-C3 = $2.247 \AA$ ) is becoming a single $\sigma$-bond. In other words the allyl chain remains connected to the palladium through $\mathrm{C} 3$ only. Also the chlorine atom and the phosphine are slightly moving far from the metal, as consequence of the incoming of the $\mathrm{CO}$ molecule. Although the addition of 
the new ligand is not sterically hindered, the activation energy associated to the process is neither negligible nor very high: $9.1 \mathrm{kcal} \mathrm{mol}^{-1}$ : especially the formation of the new C4-Pd bond requires a certain degree of electronic re-organization on the metal.

\section{(Table 10)}

(Figure 5 here)

(Scheme 7 here)

In the next intermediate, $\mathbf{M 2 3}$, the palladium has reached its typical and stable square planar tetra-coordination, which, until this point was impossible for the presence of two (or more) cumbersome triphenylphosphines in the metal coordination sphere. The other important feature of this structure is the presence, among the metal ligands, of the two groups between which the new C-C will form (i.e. the allyl group and the $\mathrm{CO}$ molecule). In particular, the distance between the two carbon atoms, $\mathrm{C} 3$ and C4, which will establish the bond, is $2.813 \AA$ A. A weak, but not negligible interaction is active between one "spectator" phosphine and the metal $(\mathrm{P} 2-\mathrm{Pd}=3.977$ $\AA$ A). Several attempts of bonding this phosphine to the palladium failed: however, since the steric hindrance is allowing it, a slight interaction is present and surely contributes to stabilize this complex, which is $4.7 \mathrm{kcal} \mathrm{mol}^{-1}$ above the AL. It is interesting to note that, all the intermediates located until now along this pathway lie more or less at the same energy level, regardless of the nature of the metal ligands (allyl, chlorine, phosphine or CO molecule), except for M19 where three cumbersome triphenylphosphines are bonded to the palladium). In $\mathbf{M 2 3}$, the palladium oxidation number is still +2 . 
(Scheme 8 here)

Before proceeding along this reaction channel, it is necessary to point out that before the CO comes into play, from M21 the system can evolve to M23 in a different way (a schematic representation of the structures of this alternative part of the reaction pathway is given in Scheme 8). The diffuse nature of the $\pi-\eta^{3}$ bonding in M21 allows an easy conversion to an $\alpha$-allyl-palladium complex in which the allyl is attached to the metal through a single $\sigma$ carbon-metal bond (C3-Pd). In this case, this transformation takes place before and not simultaneously with the $\mathrm{CO}$ approach, like it happens in TS16. In the transition state TS17 the Pd-C1 bond is clearly elongating $(\mathrm{Pd}-\mathrm{C} 1=2.890 \AA), \mathrm{C} 1-\mathrm{C} 2$ is assuming a double bond character $(\mathrm{C} 1-\mathrm{C} 2=1.365 \AA)$ and the chlorine atom is approaching the palladium $(\mathrm{Pd}-\mathrm{Cl}=2.616 \AA)$, which is being taken off part of the charge density previously donated from the allyl unit. Since only a rearrangement of the allyl-palladium bonding is taking place, the energy barrier associated to TS17 is quite modest: $3.2 \mathrm{kcal} \mathrm{mol}^{-1}$. As anticipated, the complex that forms, M24, is characterized by a $\sigma$ carbon-palladium bond and by a square-planar coordination. However, M24 is less stable than both M21 and M22 (it is located 6.7 kcal $\mathrm{mol}^{-1}$ above the AL). This is a sign of a slight bigger strength of the $\pi-\eta^{3}$ interaction over a $\sigma$-bond and of the weakness of the palladium-triphenylphosphine bond when the system is crowed and electronically saturated. The next transition state TS18 involves the approach of the $\mathrm{CO}$ molecule to the palladium and the simultaneous expulsion of one phosphine: this leads the system to the abovedescribed M23 complex. Even in this case the forming Pd-C4 bond is almost formed $\left(\mathrm{Pd}-\mathrm{C} 4=2.292 \AA\right.$ ) ) and TS18 is only $0.6 \mathrm{kcal} \mathrm{mol}^{-1}$ below TS16 (but $1.8 \mathrm{kcal} \mathrm{mol}^{-1}$ 
above at the PCM level), that is the corresponding transition state for the Pd-C4 bond formation from the $\pi-\eta^{3}$ M22 complex. In TS18 no electronic rearrangement is needed for the allyl-palladium interaction and also a phosphorous-palladium bond is being broken $(\mathrm{Pd}-\mathrm{P} 2=3.173 \AA$ A $)$. TS18 has an activation energy of $7.2 \mathrm{kcal} \mathrm{mol}^{-1}$ (this point is $13.9 \mathrm{kcal} \mathrm{mol}^{-1}$ above the reagents) confirming that the forming of the palladium-CO bond requires a certain amount of energy. An energy analysis of the two alternative paths proceeding from M21 to M23 allows us to conclude that both are possible and competitive; the differences between them are actually slight and rely basically on the timing by which the ligands are added and removed from the palladium before reaching the $\mathbf{M 2 3}$ intermediate (see also Figure 5).

(Scheme 9 here)

The transition state TS19 following M23 (see Scheme 9) is the critical point, which describes the other crucial transformation of the process: the formation of the $\mathrm{C} 3-\mathrm{C} 4$ bond. The two atoms are part of the allyl chain and the $\mathrm{CO}$ molecule, which are in cis position around the metal, so that their approach is not difficult $(\mathrm{C} 3-\mathrm{C} 4=$ $2.053 \AA$ ). The transition state is characterized by a three-cantered structure: apart from the C3-C4 approach, simultaneously, the $\mathrm{C} 4$ bond is moving closer to the palladium (Pd-C4 = $1.903 \AA)$, whereas C3 is moving away from it $(2.404 \AA)$ so that only the former will remain bonded to metal. The energy barrier related to this process is 15.8 $\mathrm{kcal} \mathrm{mol}^{-1}\left(13.3 \mathrm{kcal} \mathrm{mol}^{-1}\right.$ at the PCM level) and furthermore TS19 lies $1.7 \mathrm{kcal} \mathrm{mol}^{-1}$ (0.6 kcal mol ${ }^{-1}$ at the PCM level) lower than TS14, the highest point located on this reaction pathway. The structure of TS19 is emblematic of how the metal performs its catalytic, assisting role, by acting as a "dock" to which two groups can attach to, 
before connecting to each other. Even in this case, an equivalent transition state, in which one more phosphine is on the metal, has been located: this structure is $4.1 \mathrm{kcal}$ $\mathrm{mol}^{-1}$ less stable than TS19. Once again, even if bonded to the metal, an additional phosphine does not afford an energy stabilization. After the discussed C-C bond formation, the system evolves to an intermediate, $\mathbf{M 2 5}$, in which the palladium is bearing three ligands only: one phosphine, one chlorine atom and the allyl carbonyl chain. The $\mathrm{CO}$ unit has inserted into the carbon-palladium bond and now the metal is linked to a 4-carbon chain. In spite of the unsaturated metal coordination sphere, this complex is more stable than all the other structures determined so far, because of the formation of a new carbon-carbon bond (M25 lies $0.4 \mathrm{kcal} \mathrm{mol}^{-1}$ below the AL and 5.1 lower than the previous intermediate). At this point, there is space for an easy approach of a triphenylphosphine to the unsaturated palladium (TS20, activation energy $=0.4 \mathrm{kcal} \mathrm{mol}^{-1}$ ) that brings the system to the already discussed M18. The subsequent steps are obviously those already described in the section 3.B.1 and terminates the catalytic cycle. It is worth mentioning that a transition state for an approach of the chloroallyl unit to the palladium in the initial $\mathrm{Pd}\left(\mathrm{PPh}_{3}\right)_{3}$ complex that would eventually lead directly to its oxidation has been found. This transition state would lead to $\mathbf{M 2 4}$ (see Scheme 8), after the concerted breaking of the allylic carbonchlorine bond and the formation of the relative carbon-palladium and chlorinepalladium bonds. Additionally, one phosphine leaves the metal. Although this transition state exists, it is located very high in energy compared to any other points discussed so far: $39.6 \mathrm{kcal} \mathrm{mol}^{-1}$. It is evident that there is a high cost of energy for the system when a single carbon atom is involved in the breaking of a bond and the simultaneous forming of an interaction with the palladium. In all the cases of carbonmetal $\sigma$-bond formation described above, the carbon atom was previously involved in 
a $\pi$-interaction $\left(\eta^{2}\right.$ or $\left.\eta^{3}\right)$ with the palladium that makes smooth and quite easy the establishment of the subsequent bond.

(Table 11)

3.B.3. Formation of $\pi \eta^{2}$-allyl-palladium complexes: an alternative way to carbonylation?

The investigation of the potential surface has shown the existence of a third carbonylation pathway involving the formation of $\pi \eta^{2}$ complexes between the allyl moiety and the metal ( $\eta^{2}$ pathway). From the above-discussed M19 tetrahedral intermediate, the $\mathrm{CO}$ molecule can enter the metal coordination sphere (transition state TS21, see Scheme 10) and simultaneously interacts with the allyl chain (C1-C4 $=1.766 \AA$ ) and one of the three phosphine units $(\mathrm{C} 4-\mathrm{P} 1=2.434 \AA$ ). As a consequence of these interactions the $\mathrm{C} 1 \mathrm{C} 2$ double bond becomes much longer $(\mathrm{C} 1-\mathrm{C} 2=1.425 \AA)$ and the Pd-C2 forming is almost complete. A cyclic structure can be recognized involving the following five atoms: $\mathrm{C} 2, \mathrm{Pd}, \mathrm{P} 1, \mathrm{C} 4$ and $\mathrm{C} 1$ that forms completely in the following intermediate M26. However TS21 is $45.1 \mathrm{kcal} \mathrm{mol}^{-1}$ above the starting reagents and $22.9 \mathrm{kcal} \mathrm{mol}^{-1}$ higher than TS14 (these values become 12.3 and 30.4 kcal $\mathrm{mol}^{-1}$ when solvent effects are taken into account). M26 is also very high in energy being $35.1 \mathrm{kcal} \mathrm{mol}^{-1}$ less stable than the reagents. Thus, the $\eta^{2}$ pathway cannot compete with the $\pi-\eta^{3}$ (and not even with the $\sigma$ pathway) and will not be discussed further.

(Scheme 10) 


\section{C. Extended Basis Set (sdd/TZVP/DZVP) Results}

In this section, we discuss the results obtained at the extended sdd/TZVP/DZVP level for the following critical points: (a) M3, TS2, M4, M5, TS3, M12 and TS8 for the conversion and reduction mechanism of $\mathrm{Pd}(\mathrm{OAc})_{2}$ to $\mathrm{Pd}(0)$ complexes (Figure 1 and Figure 2); (b) M17 and TS11 for the carbonylation along the $\sigma$-pathway (Figure 4); (c) M20, TS14, TS16, TS18, M22, M23, M24 and TS19 for the carbonylation occurring along the $\pi \eta^{3}$-pathway (Figure 5); (d) TS21 i.e. the transition state for the carbonylation along the $\pi \eta^{3}$-channel. The energy values obtained at the sdd/TZVP/DZVP level are reported in square brackets in Figure 1, 2, 4 and 5.

The most important aspects evidenced by these computations (compared to the DZVP/3-21G* energetics) can be summarized as follows:

(i) $\mathrm{The} \mathrm{Pd}(\mathrm{II}) \rightarrow \mathrm{Pd}(0)$ reduction remains an easy process and the general pattern of the energy profile does not change appreciably. The trans pathway is still favored over the cis one being TS8 $15.9 \mathrm{kcal} \mathrm{mol}^{-1}$ higher in energy than TS3. Also the corresponding intrinsic energy barrier is higher for the cis pathway (55.2 $\left.\mathrm{kcal} \mathrm{mol}^{-1}\right)$ than for the trans pathway $\left(43.3 \mathrm{kcal} \mathrm{mol}^{-1}\right)$. The energy difference between TS3 and TS8 and the starting reagents becomes larger at the sdd/TZVP/DZVP level: thus both critical points are now (more realistically) above the $\mathrm{AL}$

(ii) The mechanistic pattern describing the possible reaction channels for the carbonylation of allyl chloride catalyzed by $\mathrm{Pd}\left(\mathrm{PPh}_{3}\right)_{4}$ does not change significantly. The values of the intrinsic energy barriers for the most important transition states of the $\pi-\eta^{3}$ pathway (TS14, TS16, TS18 and TS19) are very similar to those obtained with the DZVP/3-21G* basis set. The most significant difference has been found for the M22 $\rightarrow$ TS16 energy barrier, which is now $3.7 \mathrm{kcal} \mathrm{mol}^{-1}$ lower. Therefore. the 
system is likely to evolve from M21 to M23 along the TS16 channel instead of the

TS18 channel (these points were almost degenerate at the DZVP/3-21G* level).

Furthermore the $\pi-\eta^{3}$ pathway is more favored with respect to the $\sigma$-pathway: the

highest transition state found along the former pathway (TS14) lies now $10 \mathrm{kcal} \mathrm{mol}^{-1}$

lower than the highest transition state on the latter (TS11).

(iii) The transition state TS21 for the rate-determining step of the third

possible carbonylation pathway ( $\eta^{2}$-pathway), lies $45.2 \mathrm{kcal} \mathrm{mol}^{-1}$ above the AL and

$37.2 \mathrm{kcal} \mathrm{mol}^{-1}$ higher than TS14. Thus, even according to the extended basis set

computations, this channel to carbonylation cannot compete with the two above

discussed $\pi-\eta^{3}$ and $\sigma$ reaction pathways.

\section{Conclusions}

Our results can be summarized as follows:

1) The reduction process of $\mathrm{Pd}(\mathrm{II})$ to $\mathrm{Pd}(0)$ requires an excess of phosphines that react easily (low barriers and high exothermicity) with the starting reagent $\operatorname{Pd}(\mathrm{OAc})_{2}$.

2) The consecutive interaction of two phosphines with $\mathrm{Pd}(\mathrm{OAc})_{2}$ leads to the tetra-coordinated trans- $\mathrm{Pd}(\mathrm{OAc})_{2}\left(\mathrm{PPh}_{3}\right)_{2}$ species (M4) where the metal is still in the +2 oxidation state. The actual reduction goes first through an intra-molecular rearrangement where the two acetate fragments move toward the phosphorus atom of one phosphine ligand bound to the metal. Then, an additional phosphine enters the system leading to the formation of $\mathrm{Pd}\left(\mathrm{PPh}_{3}\right)_{2}$ and $\mathrm{PPh}_{3}(\mathrm{OAc})_{2}$. In the final stage, the involvement of two more phosphines affords the final product $\mathrm{Pd}\left(\mathrm{PPh}_{3}\right)_{4}$ where the oxidation state of the metal is zero. We would like to point out that our computations, because of the approximations that feature our model-system, do not completely rule 
out the conclusions reached by Amatore and Jutland [11] who suggest the involvement of ionic species. [74]

3) An alternative path leading to reduction involves the tetra-coordinated cis- $\mathrm{Pd}(\mathrm{OAc})_{2}\left(\mathrm{PPh}_{3}\right)_{2}$ complex exists, but it is highly disfavoured since it requires the overcoming of larger energy barriers.

4) The existence of three pathways leading to the carbonylation of allyl chloride catalyzed by $\mathrm{Pd}(0)$ species such as $\mathrm{Pd}\left(\mathrm{PPh}_{3}\right)_{4}$ has been demonstrated. In the $\sigma$-pathway, the first significant coordination to the palladium complex is that of CO. Then, the allyl is involved in the formation of a cyclic transition structure, where the CO molecule interacts at the same time with the allyl chain and the metal. Simultaneously the chlorine atom migrates to the palladium. This transition state (TS11, $24.9 \mathrm{kcal} \mathrm{mol}^{-1}$ above the reactants) corresponds to the rate-determining step of the process. The catalytic cycle terminates after a second migration of the chlorine atom and a final addition of a phosphine to the palladium complex. This regenerates the initial catalyst $\mathrm{Pd}\left(\mathrm{PPh}_{3}\right)_{4}$. This mechanism is very similar to that determined in a previous work for the same reaction catalyzed by $\mathrm{Pd}(\mathrm{II})$ species $\left(\mathrm{PdCl}_{2}\left(\mathrm{PR}_{3}\right)_{2}\right)$ [53].

The second pathway ( $\pi-\eta^{3}$-pathway) features the formation of $\eta^{3}$ complexes that follows the initial coordination of the allyl substrate in a $\pi \eta^{3}$ fashion. This step, which corresponds to the rate determining step of the process (TS14, $22.2 \mathrm{kcal} \mathrm{mol}^{-1}$ above the reactants), is associated with the chlorine migration to the metal that oxidizes to $\mathrm{Pd}(\mathrm{II})$. In a subsequent step the $\mathrm{CO}$ molecule also binds to palladium before insertion into the palladium-allyl bond. A second migration of the chlorine atom terminates the catalytic cycle and determines the release of the product. A final addition of a phosphine to the palladium complex leads to the regenerated catalyst $\mathrm{Pd}\left(\mathrm{PPh}_{3}\right)_{4}$. Since the transition states for the rate-determining steps of these two 
reaction channels are very close in energy, both reaction paths are likely and can compete.

Finally, a third pathway ( $\eta^{2}$-pathway) involves the CO attack on a $\pi \eta^{2}$ allylpalladium complexes, which anticipates the chlorine migration. However, in this case, the transition state of the rate-determining step (TS21) is much higher in energy than the above-mentioned transition states, so that this reaction path cannot compete with the two above described.

5) Our computations clearly indicate that, when using catalysts such as $\mathrm{Pd}(\mathrm{OAc})_{2}$ with an excess of phosphines, the real catalyst is a $\mathrm{Pd}(0)$ species.

6) During the carbonylation the Pd formal oxidation state changes from 0 to +2 and then to 0 again.

7) The inclusion of solvent effects, computed with the PCM approach, does not significantly affect the mechanistic scenario obtained from gas-phase computations

8) The chlorine atom plays a determinant role in this kind of processes, thanks to its significant migrating attitude and capacity of interacting with the metal even at long distances.

9) The cumbersome phosphines influence the course of the reaction and the stability of the various complexes: in several cases, the metal prefers to expel a phosphine in order to reduce the steric hindrance in the coordination sphere and hence allowing stronger bonds with the remaining ligands.

10) The computations at the extended sdd/TZVP/DZVP level do not significantly affect the mechanistic picture provided by the DZVP/3-21G* basis set. The intrinsic energy barriers computed for the key steps of the various reaction paths 
and the relative stability of the most important transition states change only slightly, thus confirming the conclusions reached at the less accurate level.

\begin{abstract}
Acknowledgments
Acknowledgment to HPC-Europa program: this work was carried out under the HPC-EUROPA project (RII3-CT-2003-506079), with the support of the European Community - Research Infrastructure Action under the FP6 "Structuring the European Research Area" Programme. Financial support has also been provided by the Spanish Government (Project CQTC-2008-06644C03-01) and the Generalitat de Catalunya (SGR-2009-462).
\end{abstract}

Table 1. Selected bond lengths ( $⿱$ ) for the critical points M0, M1, TS1, M2 and M3.

Table 2. Selected bond lengths $(\AA)$ for the critical points TS2, M4 and M5

Table 3. Selected bond lengths ( $\AA$ ) for the critical points TS3, M6, TS4 and M7

Table 4. Selected bond lengths $(\AA)$ for the critical points M8, TS5, M9, M10, TS6 and M11.

Table 5. Selected bond lengths ( $\AA$ ) for the critical points TS7, M12 and TS8.

Table 6. Selected bond lengths (Å) for the critical points M13, M14, TS9 and M15. Table 7. Selected bond lengths ( $)$ ) for the critical points M16, TS10, M17, TS11, M18 and TS12.

Table 8. Selected bond lengths $(\AA)$ for the critical points M19, TS13, M20.

Table 9. Selected bond lengths ( $\AA$ ) for the critical points TS14, M21, TS15 and M22. Table 10. Selected bond lengths $(\AA)$ for the critical points TS16, M23, TS17, M24 and TS18

Table 11. Selected bond lengths ( $)$ ) for the critical points TS19, M25 and TS20

Figure 1. Energy profile of the addition of two tri-phenylphosphine units to the starting $\mathrm{Pd}(\mathrm{OAc})_{2}$ complex. Energy values are in $\mathrm{kcal} \mathrm{mol}^{-1}$. PCM values in round brackets and sdd/TZVP/DZVP values in square brackets. The position of the $\mathrm{x}$ axis (energy of asymptotic limit) has been set according to the DZVP/3-21G* values. Figure 2. Energy profile of the trans- and cis-channel (dashed line) for the palladium reduction process. Energy values are in $\mathrm{kcal} \mathrm{mol}^{-1}$. PCM values in round brackets and sdd/TZVP/DZVP values in square brackets. The position of the $\mathrm{x}$ axis (energy of asymptotic limit) has been set according to the DZVP/3-21G* values.

Figure 3. Energy profile of the $\sigma$-pathway for the carbonylation reaction of allyl chloride catalyzed by $\mathrm{Pd}(0)$ complexes. Energy values are in $\mathrm{kcal} \mathrm{mol}^{-1}$. PCM values in round brackets.

Figure 4. Energy profile of the $\sigma$-pathway for the carbonylation reaction of allyl chloride catalyzed by $\mathrm{Pd}(0)$ complexes. Energy values are in $\mathrm{kcal} \mathrm{mol}^{-1}$. PCM values in round brackets and sdd/TZVP/DZVP values in square brackets. The position of the $\mathrm{x}$ axis (energy of asymptotic limit) has been set according to the DZVP/3-21G* values.

Figure 5. Energy profile of the $\pi \eta^{3}$-pathway for the carbonylation reaction of allyl chloride catalyzed by $\operatorname{Pd}(0)$ complexes. In dashed line (and italic for lettering), the second possible pathway connecting M21 to M23. Energy values are in $\mathrm{kcal} \mathrm{mol}^{-1}$. PCM values in round brackets and sdd/TZVP/DZVP values in square brackets. The 
position of the $\mathrm{x}$ axis (energy of asymptotic limit) has been set according to the $\mathrm{DZVP} / 3-21 \mathrm{G}^{*}$ values.

Scheme 1. Representation of the structure of the critical points localized on the profile of the addition of two tri-phenylphosphine units to the starting $\mathrm{Pd}(\mathrm{OAc})_{2}$ complex

Scheme 2. Representation of the structures located along trans-channel (first part) for the palladium reduction process

Scheme 3. Representation of the structures located along trans-channel (second part) for the palladium reduction process

Scheme 4. Representation of the structures located along the first part of the cischannel for the palladium reduction process

Scheme 5. Representation of the structure of the critical points located in the path for the formation of the phosphine oxide

Scheme 6. Representation of the structure of the critical points located along the $\sigma$ pathway for the carbonylation reaction of allyl chloride catalyzed by $\operatorname{Pd}(0)$ complexes Scheme 7. Structures of the critical points located along the first part of the $\pi \eta^{3}$ pathway for the carbonylation reaction of allyl chloride catalyzed by $\operatorname{Pd}(0)$ complexes Scheme 8. Representation of the structure of the critical points located along an alternative channel for passing from M21 to M25 along the $\pi \eta^{3}$-pathway for the carbonylation reaction of allyl chloride catalyzed by $\mathrm{Pd}(0)$ complexes

Scheme 9. Representation of the structure of the critical points located along the second part of the $\pi \eta^{3}$-pathway for the carbonylation reaction of allyl chloride catalyzed by $\operatorname{Pd}(0)$ complexes

Scheme 10. Representation of the structures of the critical points located on the $\eta^{2}$ pathway

[1] N. Miyaura, A. Suzuki, Chem. Rev. 95, 2457 (1995).

[2] R. F. Heck, Org. React. 27, 345 (1982).

[3] B. M. Trost, D. L. Van Vranken, Chem. Rev. 96, 395 (1996).

[4] E. I. Negishi, Acc. Chem. Res. 15340 (1982). M. Kalek, J. Stawinski, Organometallics 26, 5840 (2007).

[5] G. Kiss, Chem. Rev. 101, 3435 (2001): refs. 13k, q, s, 14b, 20f and 49 therein.

[6] J. F. Knifton, J. Am. Oil. Chem. Soc. 55, 496 (1978).

[7] D. M. Fenton, J. Org. Chem. 383192 (1973).

[8] K. Bittler, N. Kutepow, D. Neubauer, H. Reis, Angew. Chem. Int. Ed. Engl. 7, 329 (1968).

[9] E. I. Neghishi, A.O. King, N.J. Okukado, Org. Chem. 42, 1821 (1977). J. F. Fauvarque, A. Jutand, Bull. Soc. Chim. Fr. 765 (1976).

[10] C. Amatore, M. Azzabi, A.J. Jutand, Organomet. Chem. 363, C41 (1989).

[11] C. Amatore, A. Jutand, M.A. M'Barki, Organometallics 11, 3009 (1992). C. Amatore, E. Carré, A. Jutand, M.A. M'Barki, Organometallics 14, 1818 (1995). C. Amatore, A. Jutand, J. Organomet. Chem. 576, 254 (1999).

[12] C. Amatore, M. Azzabi, A. Jutand, J. Am. Chem. Soc 113, 8375 (1991). J.K. Kochi, Organometallic Mechanisms and Catalysis, Academic Press: New York 1978, Part I, Chapter 7. C. Amatore, M. Azzabi, A. Jutand, J. Am. Chem. Soc. 113, 1670 (1991). C. Amatore, A. Jutand, F. Khalil, M.A. M'Barki, L. Mottier, Organometallics 12, (1993) 3168. A.L. Casado, P. Espinet, J. Am. Chem. Soc. 120, 8978 (1998). A.L. Casado, P. Espinet, Organometallics 17, 954 (1998). P. Espinet, A. M. Echavarren, Angew. 
Chem., Int. Ed. 43, 4704 (2004). F. M. Bickelhaupt, T. Ziegler, P.v.R.

Schleyer, Organometallics 14, 2288 (1995). A. Diefenbach, F.M. Bickelhaupt, J. Chem. Phys. 115, 4030 (2001). K. Albert, P. Gisdakis, N. Rösch, Organometallics 17, 1608 (1998). M. Jakt, L. Johannissen, H.S. Rzepa, D.A. Widdowson, A. Wilhelm, J. Chem. Soc., Perkin Trans. 2, 576 (2002). A. Sundermann, O. Uzan, J. M. L. Martin, Chem. Eur. J. 7, 1703 (2001). M.H. Senn, T. Ziegler, Organometallics 23, 2980 (2004). L.J. Goosen, D. Koley, H. Hermann, W. Thiel, Chem. Commun. 2141 (2004).

[13] S. Kozuch, C. Amatore, A. Jutand, S. Shaik, Organometallics 24, 2319 (2005).

[14] C. Amatore, E. Carré, A. Jutand, M.A. M'Barki, G. Meyer, Organometallics 14, 5605 (1995). C. Amatore, A. Jutand, Acc. Chem. Res. 33, 314 (2000).

[15] S. Kozuch, S. Shaik, C. Amatore, A. Jutand, Chem. Eur. J. 10, 3072 (2004).

[16] F. Ozawa, A. Kubo, T. Hayashi, Chem. Lett. 2177 (1992).

[17] W.A. Hermann, J. Organomet. Chem. 383, 21 (1990).

[18] G. Kiss, Chem. Rev. 101, 3435 (2001).

[19] J. A. Osborn, J. F. Young, G. Wilkinson, J. Chem. Soc., Chem. Commun. 17 (1965). R.F. Heck, Palladium Reagents in Organic Synthesis, Academic Press, New York 1985. - J. Tsuji, Organic Synthesis and Palladium Compounds, Springer, Berlin 1980.

[20] H.M. Colquhoun, D.J. Thompson, M.V. Twigg, Carbonylation: Direct Synthesis of Carbonyl Compounds, Plenum Press, New York, (1991).

[21] M. Beller, B. Cornilis, C.D. Frohning, C.W. Kohlpaintner, J. Mol. Cat. 104, 17 (1995).

[22] G.P. Chiusoli, Chim. Ind. 41, 503 (1959).

[23] J. Tsuji, M. Morikawa, J. Kiji, Tetrrahedron Lett. 1811 (1963).

[24] F. Joo, H. Alper, Organometallics 4, 1775 (1985). S-I. Murahashi, Y. Imada, Y. Taniguchi, S. Higashiura, Tetrahedron Lett. 29, 4945 (1988).

[25] A. Yamamoto, Bull. Chem. Soc. Jpn. 68, 433 (1995).

[26] H. Jiang, Y. Xu, S. Liao, D. Yu, H. Chen, X. Li, J. Mol. Cat. A: Chemical 130, 79 (1998).

[27] F. Bertoux, E. Monflier, Y. Castanet, A. Mortreux, J. Mol. Cat. A: Chemical 143, 11 (1999).

[28] R. Grigg, A. Liu, D. Shaw, S. Suganthan, D.E. Woodall, G. Yoganathan, Tetrrahedron Lett. 41, 7125 (2000).

[29] M.M. Bio, L. Leighton, Organic Letters 2, 2905 (2000).

[30] S. El Houssame, L. El Firdoussi, S. Allaoud, A. Karim, Y. Castanet, A. Mortreux, J. Mol. Cat. A: Chemical 168, 15 (2001).

[31] G.P. Chiusoli, Angew. Chem. 72, 74 (1960).

[32] R.F. Heck, J. Am. Chem. Soc. 85 (1963) 2013.

[33] G.P. Chiusoli, L. Cassar, Angew. Chem. Int. Ed. Engl. 6, 124 (1967).

[34] R.F. Heck, Acc. Chem. Res. 2, 10 (1969).

[35] G.P. Chiusoli, Acc. Chem. Res. 6, 422 (1973).

[36] E. J. Kuhlmann, J. J. Alexander, Coordination Chemistry Reviews 33, 195 (1980).

[37] G. Garcia-Gomez, J.M. Moreto, J. Am. Chem. Soc. 121, 878 (1999).

[38] J. R. Johnson, P.S. Tully, P.B. Mackenzie, M. Sabat, J. Am. Chem. Soc. 113, 6172 (1991). B.A. Grisso, J.R. Johnson, P.B. Mackenzie, J. Am. Chem. Soc. 114, 5160 (1992).

[39] W.T. Dent, R. Long, G.H. Whitfield, J. Chem Soc. 1588 (1964). R. Long, G.H. Whitfield, J. Chem Soc. 1852 (1964). 
[40] J. Tsuji, J. Kiji, S. Imamura, M. Morikawa, J. Am. Chem. Soc. 86, 4350 (1964). J. Tsuji, Acc. Chem. Res. 2, 144 (1969).

[41] D. Mllstein, Organometallics 1, 888 (1982).

[42] J. Tsuji, K. Sato, H. Okumoto, J. Org. Chem. 49, 1341 (1984).

[43] S-I. Murahashi, Y. Imada, Y. Taniguchi, S. Higashiura, Tetrahedron Lett. 29, (1988) 4945.

[44] J. Kiji, T. Okano, Y. Higashimae, Y. Fukui, Bull. Chem. Soc. Jpn. 69, 1029 (1996).

[45] H. Jiang, Y. Xu, S. Liao, D. Yu, H. Chen, X. Li, J. Mol. Cat. 130, 79 (1998).

[46] F. Bertoux, E. Monflier, Y. Castanet, A. Montreux, J. Mol. Cat. 143, 11 (1999).

[47] S. El Houssame, L. El Firdoussi, S. Allaoud, A. Karim, Y. Castanet, A. Mortreux, J. Mol. Cat. A: Chem. 168, 15 (2001).

[48] J. G. Knight, K. Tchabanenko, Tetrahedron 58, 6659 (2002).

[49] R. Song, J. Zeng, B. Zhong, Catalysis Lett. 82, 89 (2002).

[50] T. Okano, N. Okabe, Bull. Chem. Soc. Jpn. 65, 2589 (1992).

[51] F. Bernardi, A. Bottoni, M. Nicastro, I. Rossi, J.J. Novoa, X. Prat-Resina, Organometallics 19, 2170 (2000).

[52] A. Bottoni, G.P. Miscione, G. P., J.J. Novoa, X. Prat-Resina, J. Am. Chem. Soc. 125, 10412 (2003).

[53] M.A. Carvajal, G.P. Miscione, J.J. Novoa, A. Bottoni, Organometallics 24, 2086 (2005).

[54] Gaussian 03, Revision C.02, M. J. Frisch, G.W. Trucks, H.B. Schlegel, G.E. Scuseria, M.A. Robb, J.R. Cheeseman, J.A. Montgomery, Jr., T. Vreven, K.N. Kudin, J.C. Burant, J.M. Millam, S.S. Iyengar, J. Tomasi, V. Barone, B. Mennucci, M. Cossi, G. Scalmani, N. Rega, G.A. Petersson, H. Nakatsuji, M. Hada, M. Ehara, K. Toyota, R. Fukuda, J. Hasegawa, M. Ishida, T. Nakajima, Y. Honda, O. Kitao, H. Nakai, M. Klene, X. Li, J.E. Knox, H.P. Hratchian, J.B. Cross, V. Bakken, C. Adamo, J. Jaramillo, R. Gomperts, R.E. Stratmann, O. Yazyev, A.J. Austin, R. Cammi, C. Pomelli, J.W. Ochterski, P.Y. Ayala, K. Morokuma, G.A. Voth, P. Salvador, J. J. Dannenberg, V. G. Zakrzewski, S. Dapprich, A.D. Daniels, M.C. Strain, O. Farkas, D.K. Malick, A.D. Rabuck, K. Raghavachari, J.B. Foresman, J.V. Ortiz, Q. Cui, A.G. Baboul, S. Clifford, J. Cioslowski, B.B. Stefanov, G. Liu, A. Liashenko, P. Piskorz, I. Komaromi, R. L. Martin, D. J. Fox, T. Keith, M.A. Al-Laham, C.Y. Peng, A. Nanayakkara, M. Challacombe, P. M. W. Gill, B. Johnson, W. Chen, M.W. Wong, C. Gonzalez, and J. A. Pople, Gaussian, Inc., Wallingford CT, (2004).

[55] A. D. Becke, J. Chem. Phys. 98, 5648 (1993).

[56] [a] G. A. Di Labio, D. A. Pratt, J. S. Wright, Chem. Phys. Lett. 297, 181 (1998). [b] J.S. Wright, E. R. Johnson, G.A. Di Labio, J. Am. Chem. Soc. 123, 1173 (2001).

[57] N. Godbout, D.R. Salahub, J. Andzelm, E. Wimmer, Can. J. Chem. 70, 5600 (1992).

[58] S. Miertus, E. Scrocco, J. Tomasi, Chem. Phys. 55, 117 (1981). S. Miertus, J. Tomasi, Chem. Phys. 65, 239 (1982).

[59] D. Andrae, U. Haeussermann, M. Dolg, H. Stoll, H. Preuss, Theor. Chim. Acta 77, 123 (1990)

[60] A. Schaefer, H. Horn, and R. Ahlrichs, J. Chem. Phys., 97, 2571 (1992); A. Schaefer, C. Huber, and R. Ahlrichs, J. Chem. Phys., 100, 5829 (1994). 
[61] A. J. Blake, R.W. Cockamn, E.A.V. Ebsworth, S.G.D. Henderson, J. H. Holloway, N.J. Pilkington, D. W. H. Rankin, Phosphorus and Sulfur, 30, 143 (1987).

[62] [a] M.A. Carvajal, G.P. Miscione, A. Accardi, J.J. Novoa, A. Bottoni, Molecular Physics 104, 805 (2006). [b] M.A. Carvajal, G.P. Miscione, A. Accardi, J.J. Novoa, A. Bottoni, Journal of Organometallic Chemistry 691, 4498 (2006). [c] F. Bernardi, G.P. Miscione, A. Bottoni, Organometallics 20, 2751 (2001).

[63] L. J. Goossen, D. Koley, H.L. Hermann, W. Thiel, Organometallics, 24, 2398 (2005).

[64] A.A.C. Braga, G. Ujaque, F. Maseras, Organometallics, 25, 3647 (2006).

[65] A. Ariafard, Z. Lin, Organometallics, 25, 4030 (2006).

[66] M. Ahlquist, P.-O. Norrby, Organometallics, 26, 550 (2007).

[67] P. Surawatanawong, M.B. Hall Organometallics, 27, 6222 (2008).

[68] R. Martin, S.L. Buchwald, Acc. Chem. Res. 41, 1461 (2008).

[69] M. Catellani, E. Motti, N. Della CA', Acc. Chem. Res. 41, 1512 (2008).

[70] G.C. Fu, Acc. Chem. Res. 41, 1555 (2008).

[71] E. Zuidema, C. Bo, P.W.N.M. van Leeuwen, J. Am. Chem. Soc. 129, 3989 (2007).

[72] X. Zhang, Z. Lu, C. Fu and S. Ma, Org. \& Biomol.Chem. 7, 3258 (2009).

[73] J. Takaya, K. Sangu K, N. Iwasawa Angew. Chem.-Int. Ed. 48, 7090 (2009).

[74] In order to verify the hypothesis that the reaction follows a pathway involving ionic species [11], we have carried out PCM calculations on the two following systems: a) $\mathrm{PPh}_{3}(\mathrm{OAc})_{2}+\mathrm{Pd}\left(\mathrm{PPh}_{3}\right)_{2}(\mathbf{M} 7)$ and the alternative charged system b) $\mathrm{PPh}_{3}(\mathrm{OAc})^{(+)}+\mathrm{Pd}\left(\mathrm{PPh}_{3}\right)_{2}(\mathrm{OAc})^{(-)}$which would form in the case of a ionic pathway. The solvent emulated by the PCM calculations was nitromethane ( $\varepsilon$ $=38.2$ ). The computational results show that system a) is $29.6 \mathrm{kcal} \mathrm{mole}^{-1}$ more stable than system b). Also, we have compared, in the same medium, the relative stability of $\mathrm{PPh}_{3}(\mathrm{OAc})_{2}$ and $\mathrm{PPh}_{3}(\mathrm{OAc})^{+}+(\mathrm{OAc})^{-}$. Even in this case the neutral system turns out to be more stable (by $6.4 \mathrm{kcal} \mathrm{mol}^{-1}$ ) than the charged one.

The above described computational findings suggest that, at least for the present model, the mechanism proposed in our study seems to be favoured with respect to a reaction pathway involving ionic species. However, it is possible, even if unlikely if we consider the significant energy difference between system a) and system b), that the approximations of an implicit solvent model (such as PCM) affect this conclusion and that the employment of a larger model system (possibly with explicit solvent description) may lead to different results. 
A (kcal mol-1) $^{-1}$

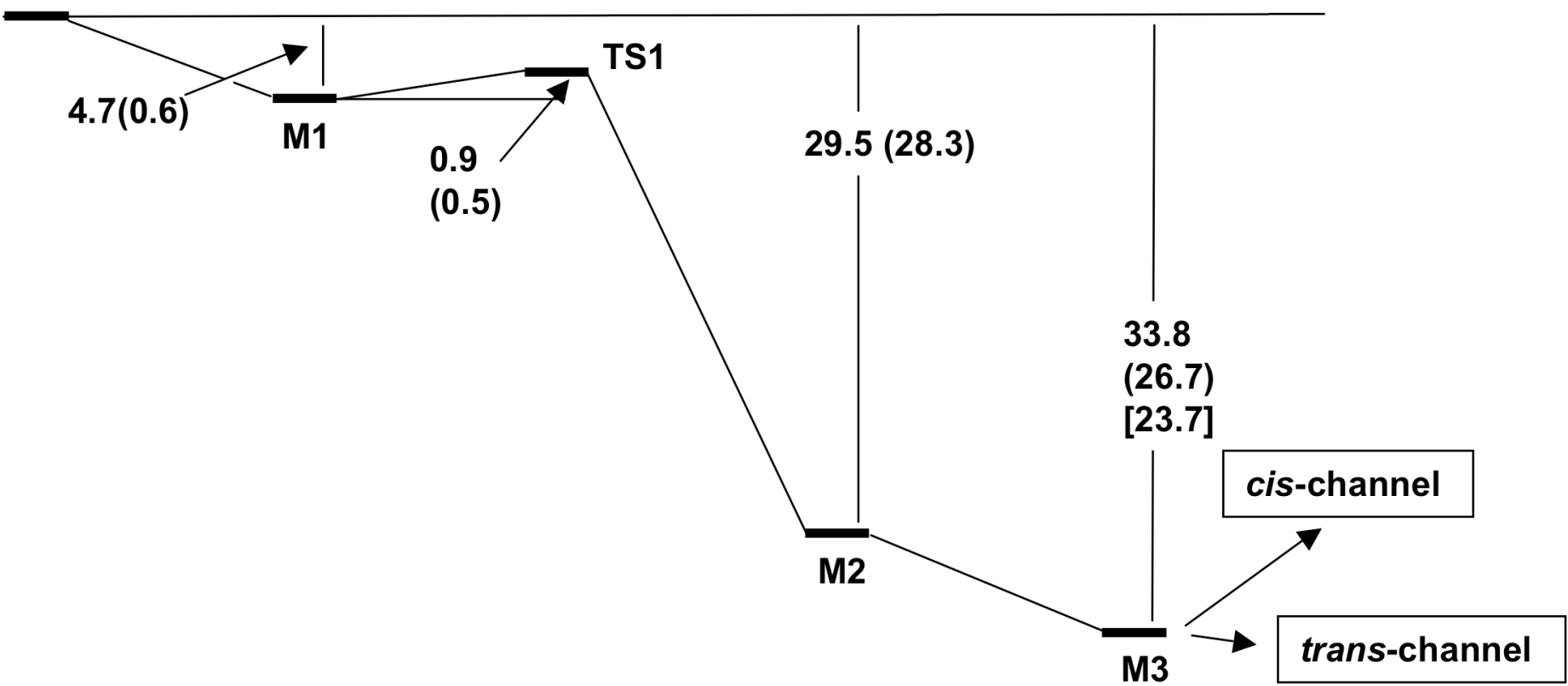




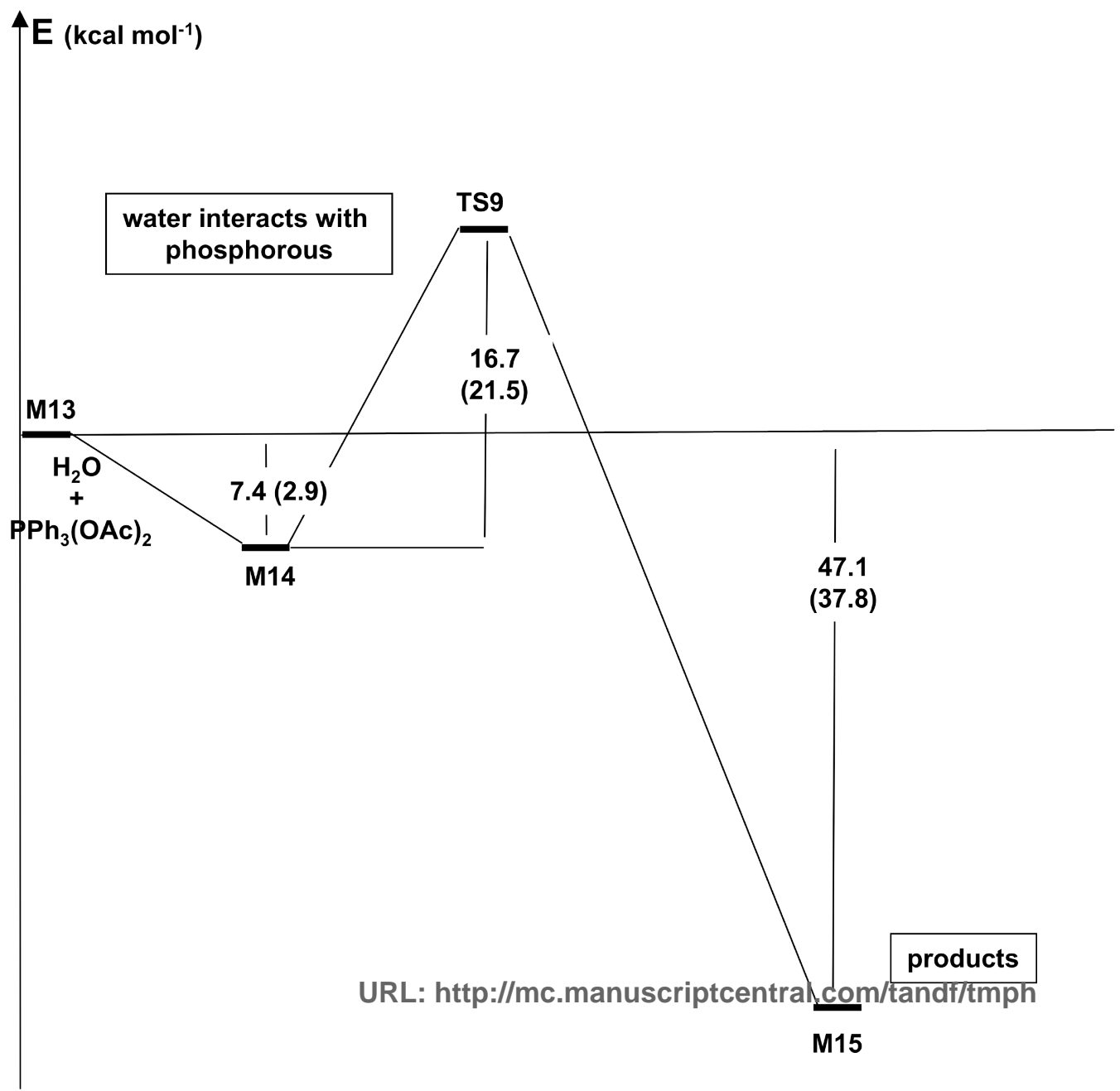




\section{Page 41 of 69

个 E (kcal mol-1)

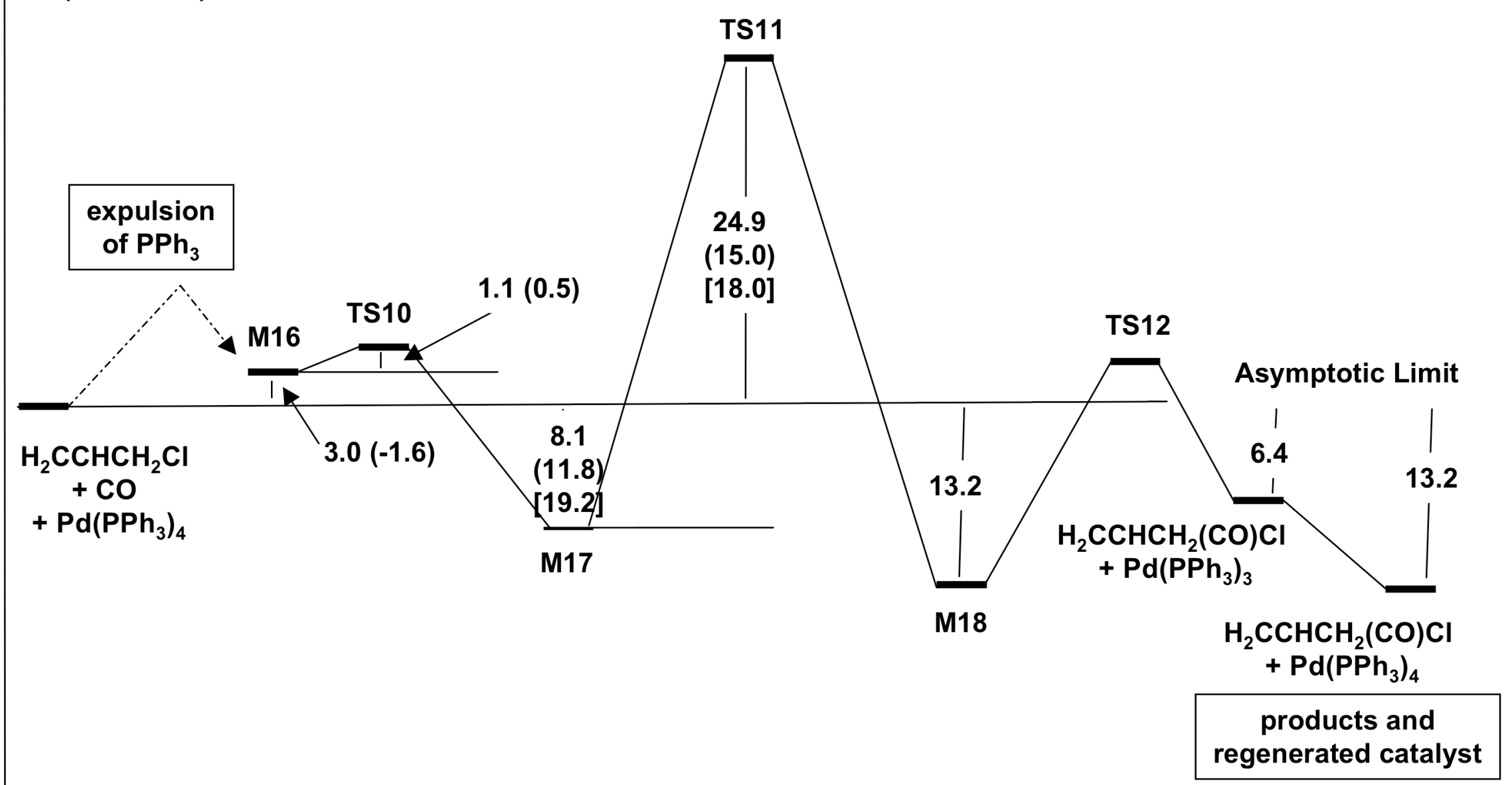




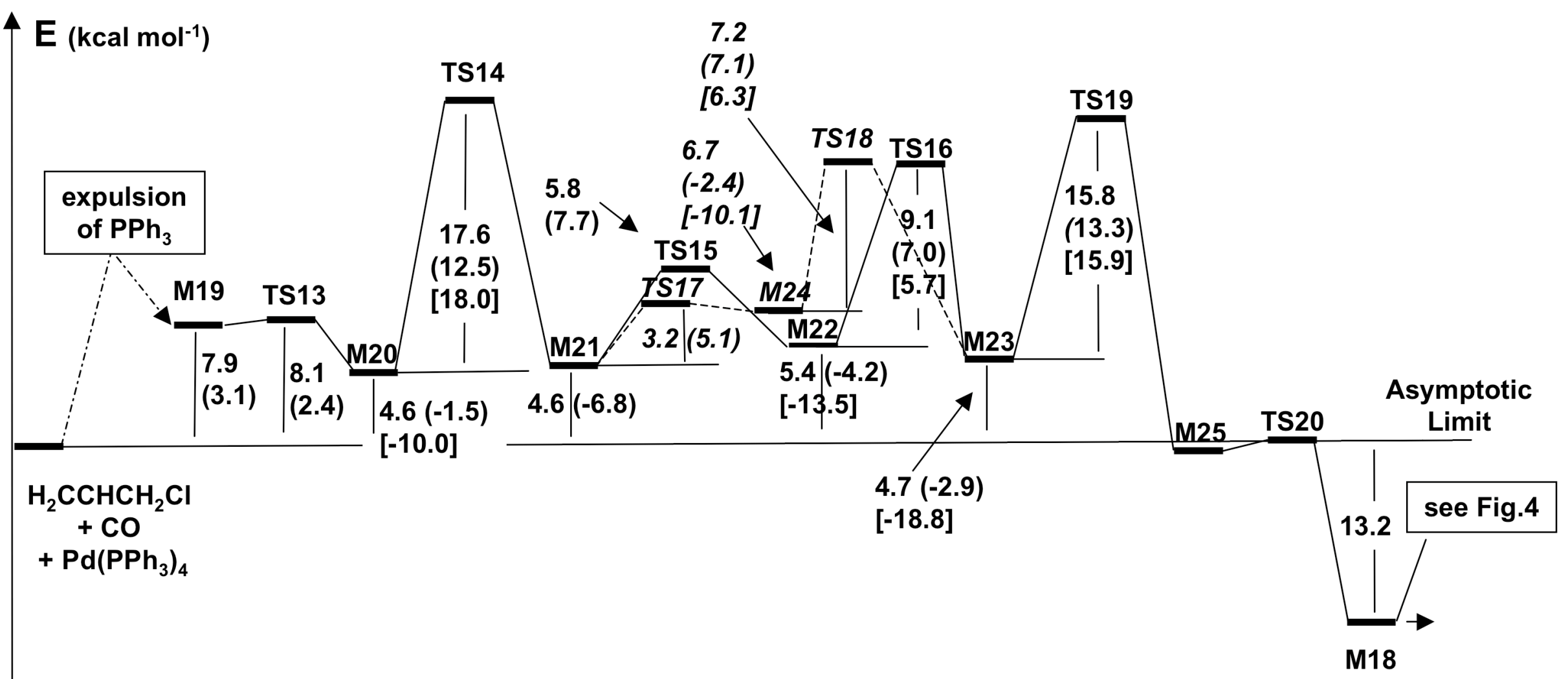




\section{Page 43 of 69}

Molecular Physics
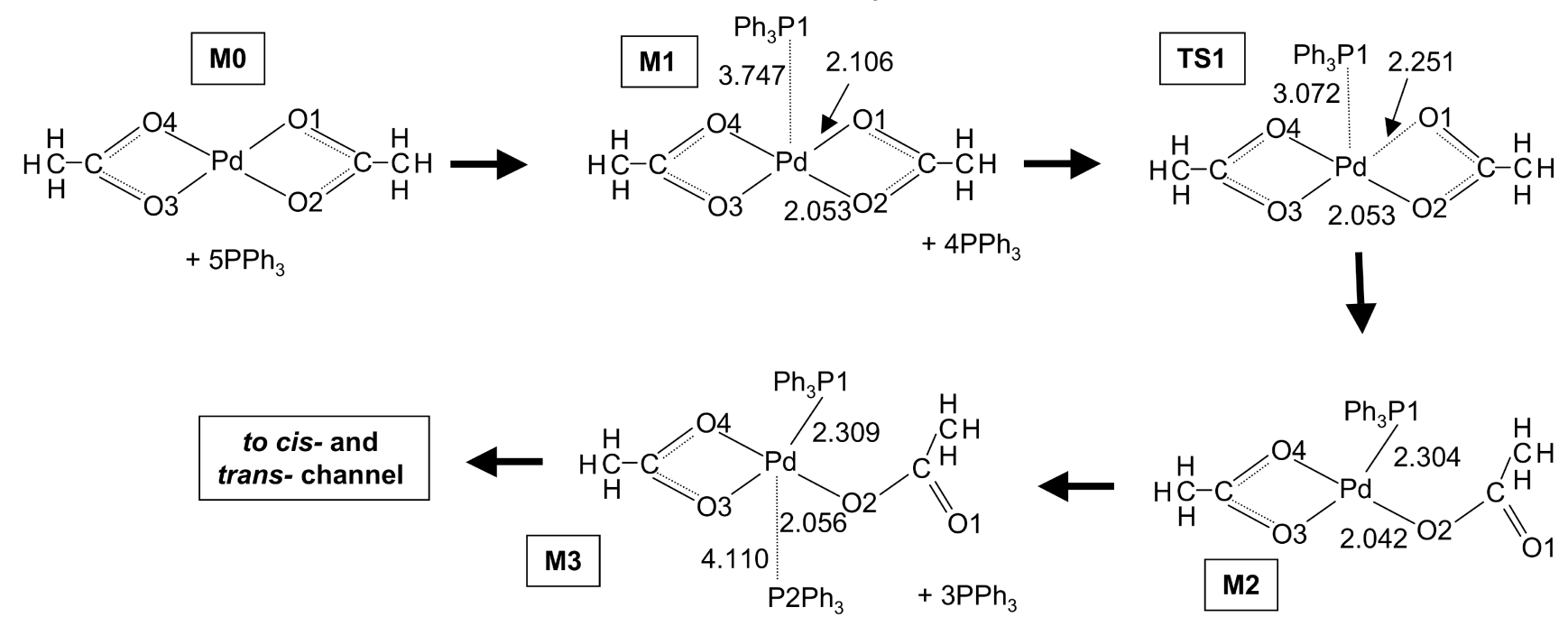

URL: http://mc.manuscriptcentral.com/tandf/tmph 


\section{trans channel}

1

2

3

4

5

6

7

8

9

10

11

12

13

14

15

16

17

18

19

20

21

22

23

24

25

26

27

28

29

\section{TS2}

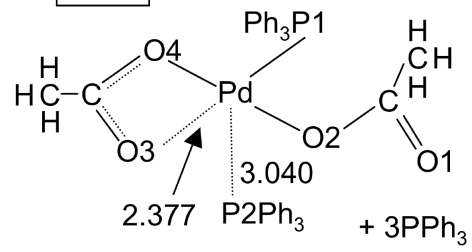

$\mathrm{E}_{\mathrm{a}}=4.5(4.1)$

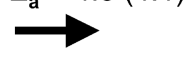

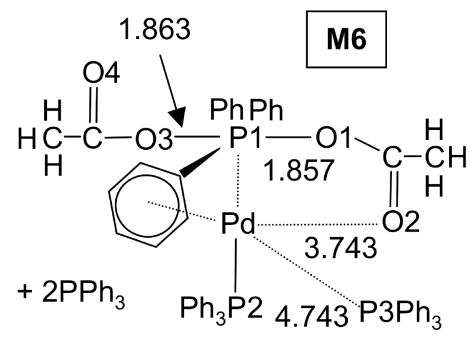
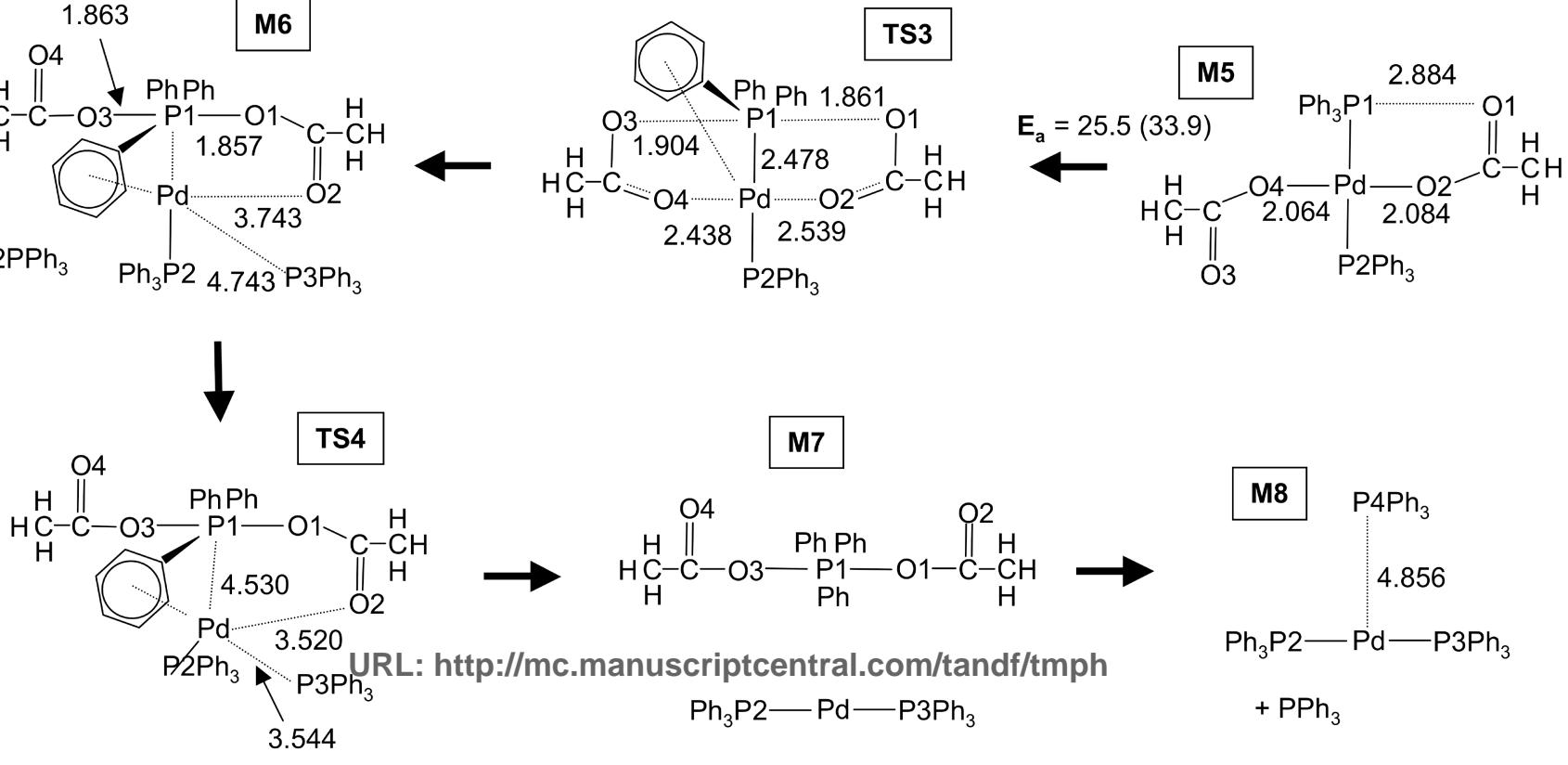

\section{M4}

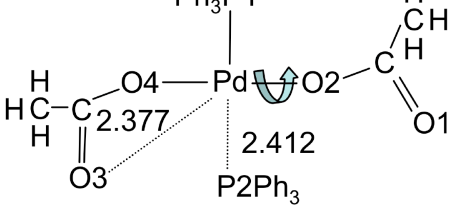

1

30 


\section{Page 45 of 69}

\section{trans channel}

1

2

3

3

4

5

6

7

8

9

10

11

12

13

14

15

16

17

18

19
Molecular Physics
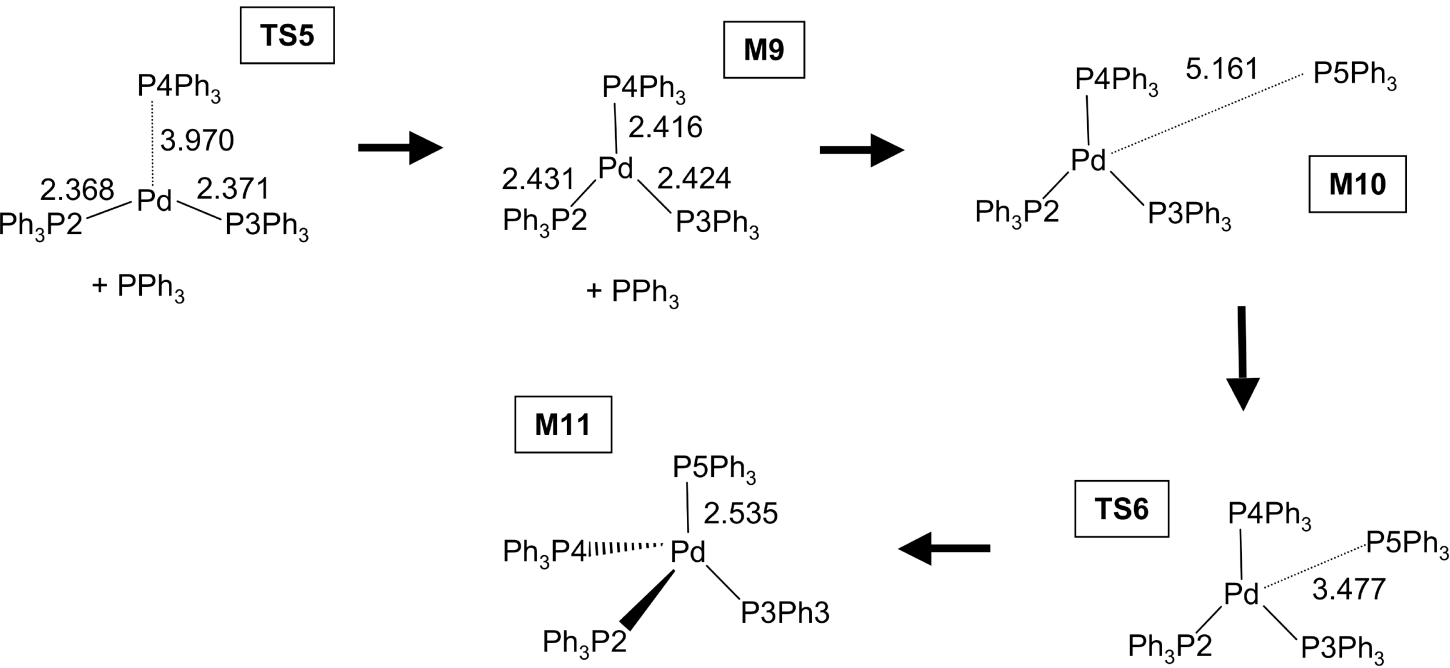

URL: http://mc.manuscriptcentral.com/tandf/tmph 


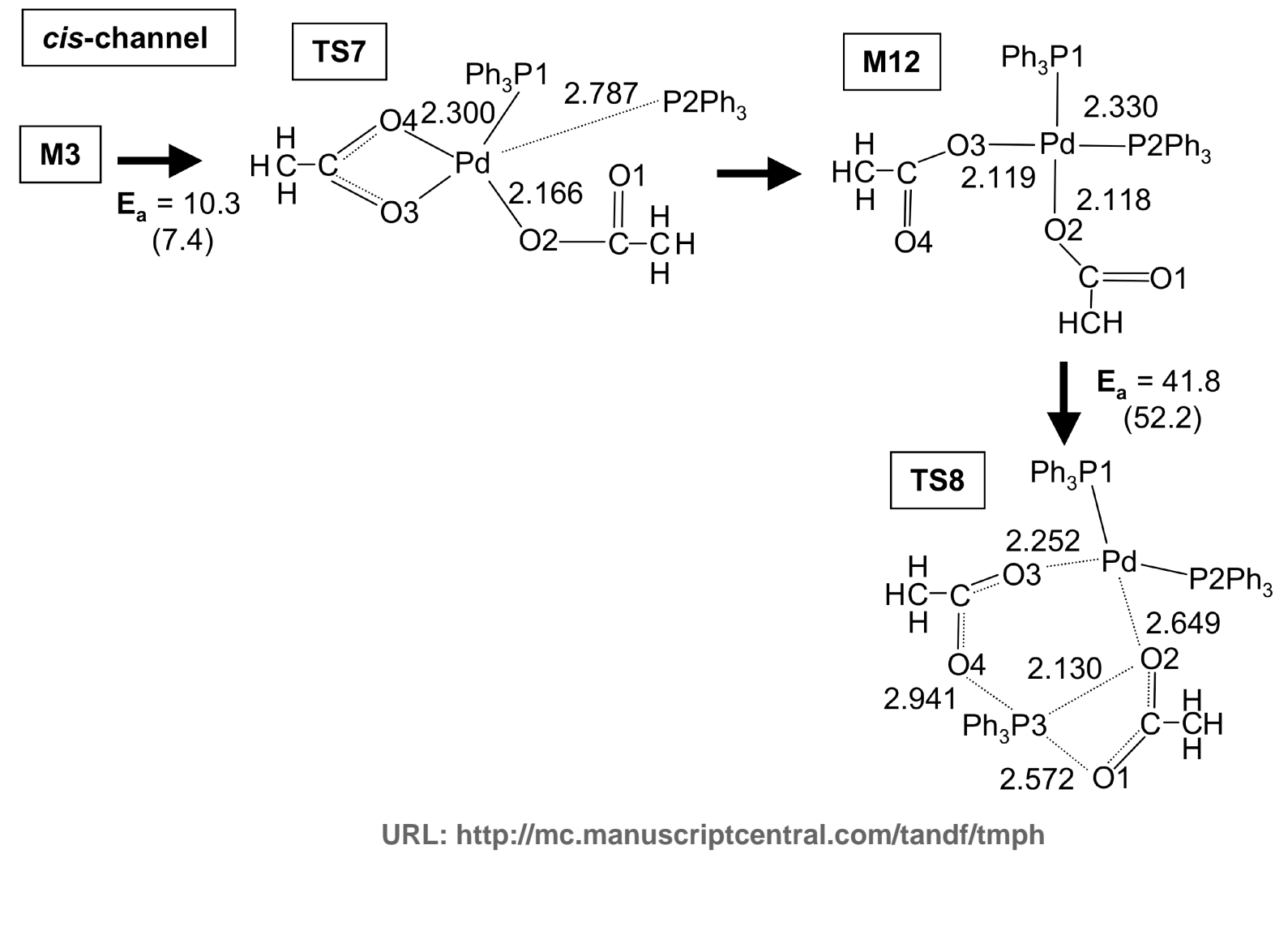




\section{Page 47 of 69}

Molecular Physics

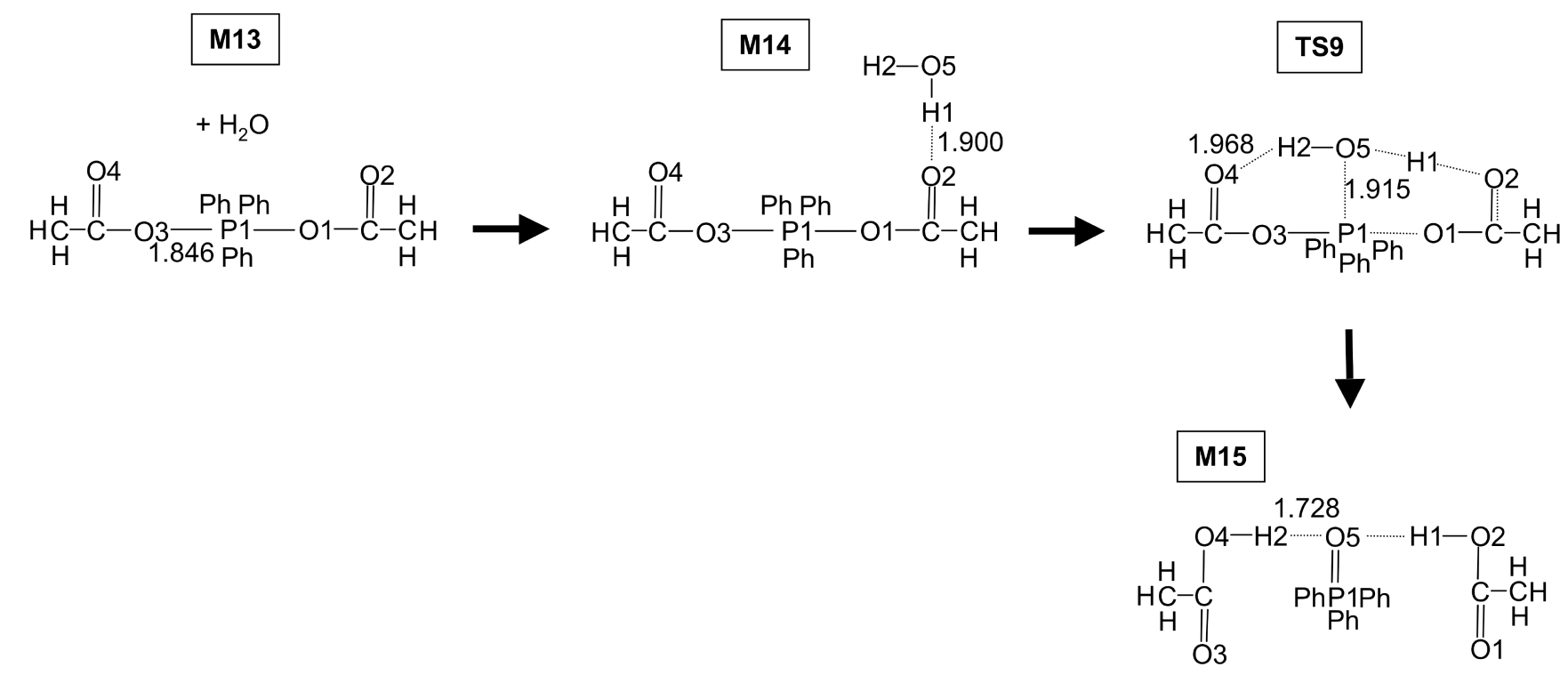

URL: http://mc.manuscriptcentral.com/tandf/tmph 
1

$\mathrm{H}_{2} \mathrm{CCHCH}_{2} \mathrm{Cl}$
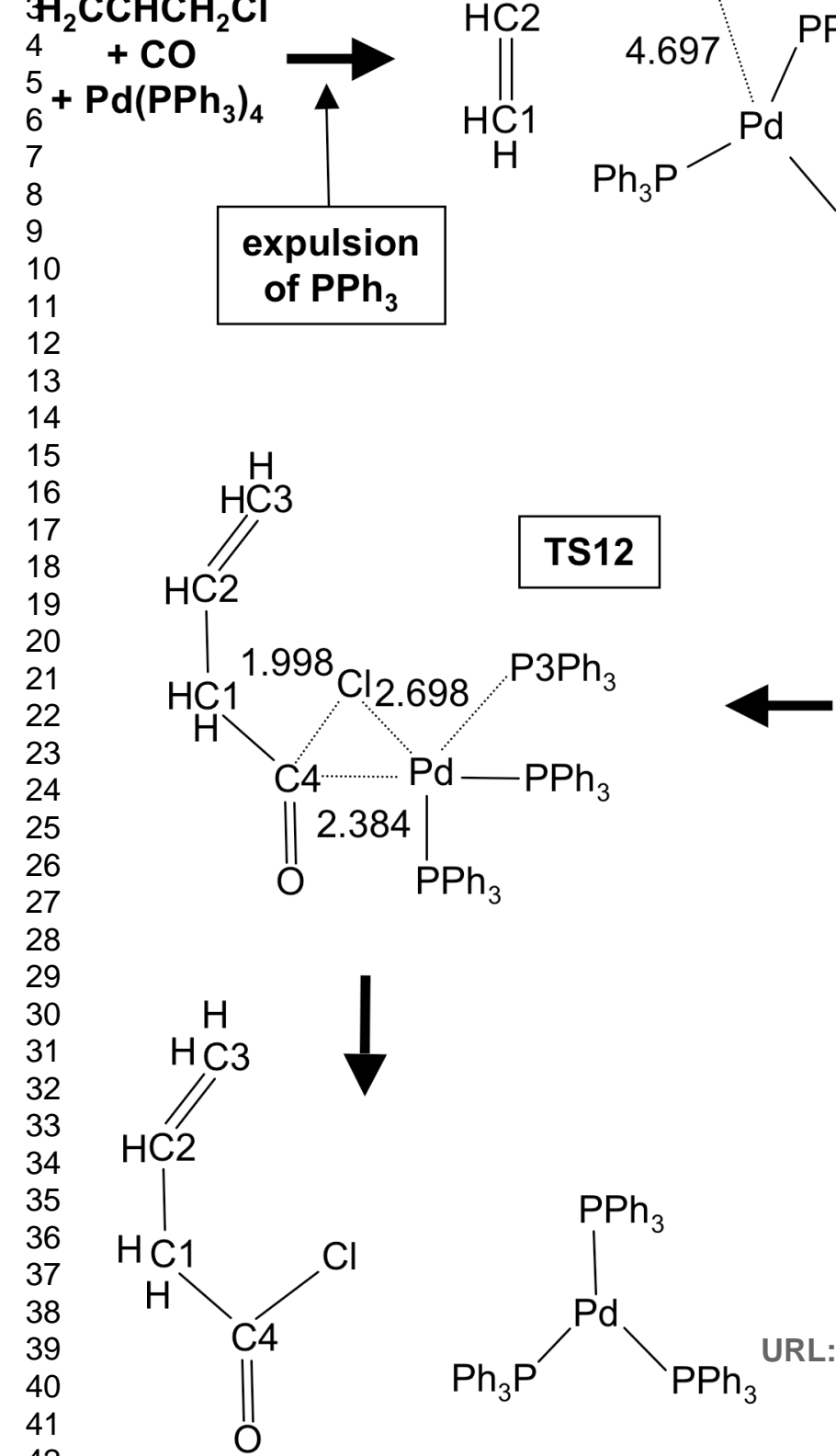

Molecular Physics
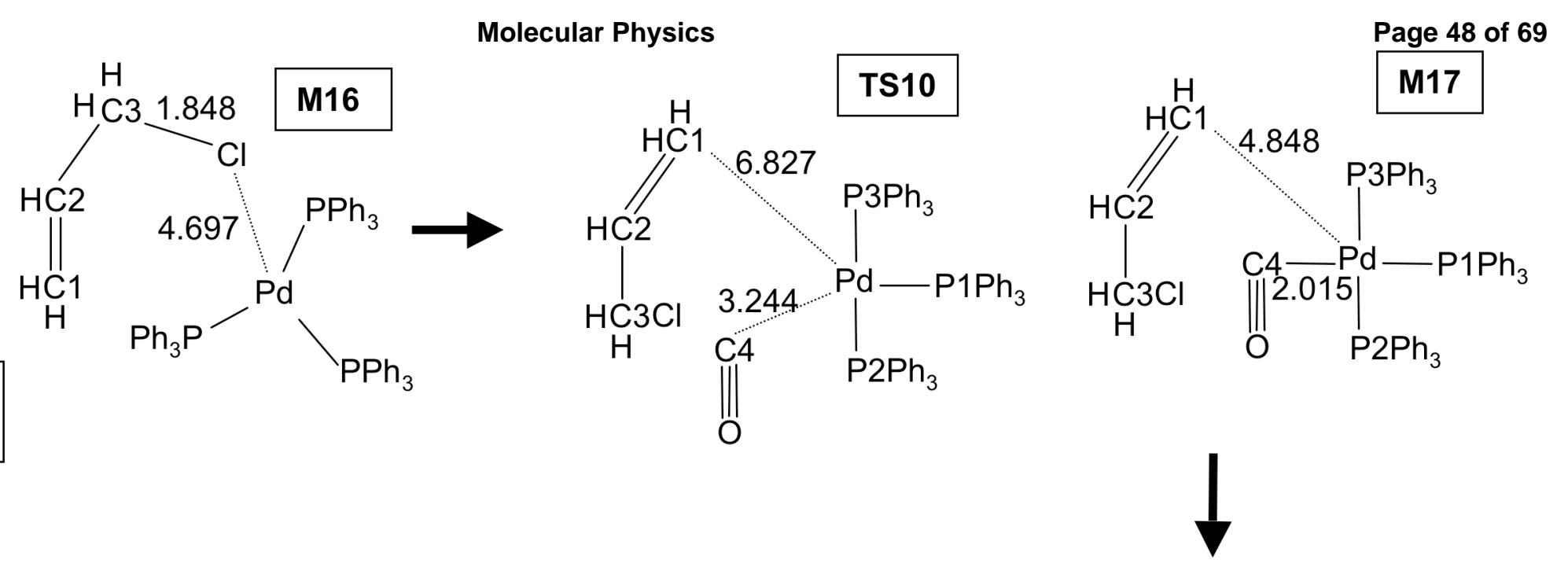

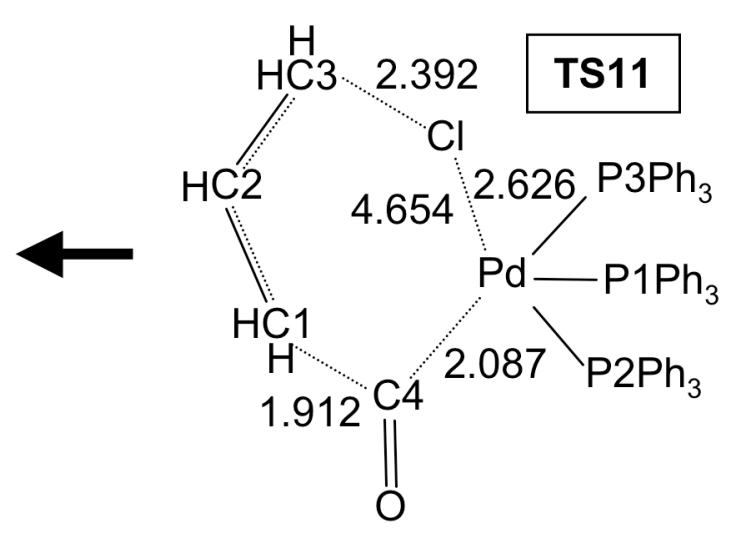

Products

\section{$\mathrm{H}_{2} \mathrm{CCHCH}_{2}(\mathrm{CO}) \mathrm{Cl}$}

$+\mathrm{Pd}\left(\mathrm{PPh}_{3}\right)$

URL: http://mc.manuscriptcentral.com/tand $/ 4$ tmph 


\section{Page 49 of 69}

\section{Molecular Physics}
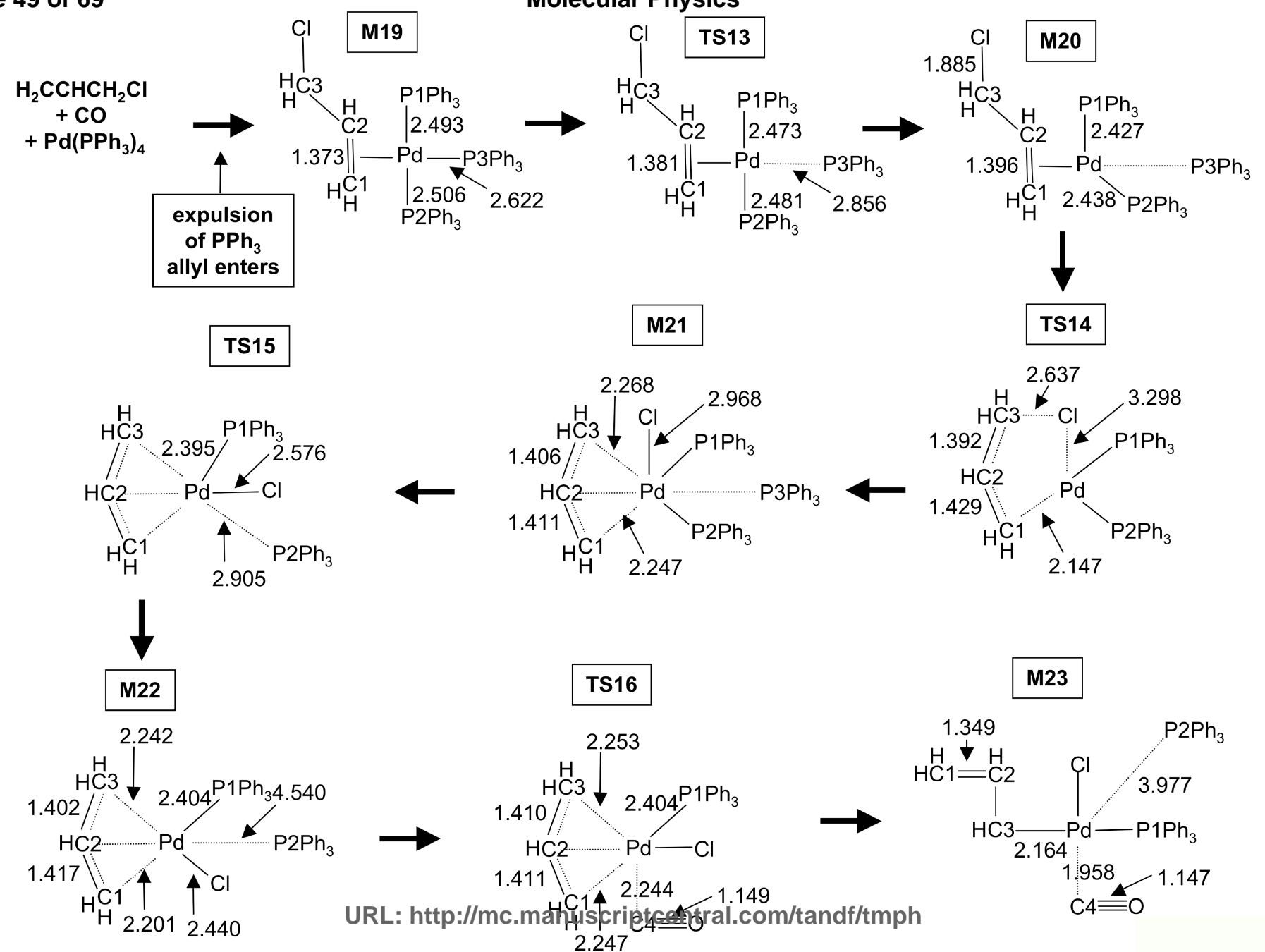


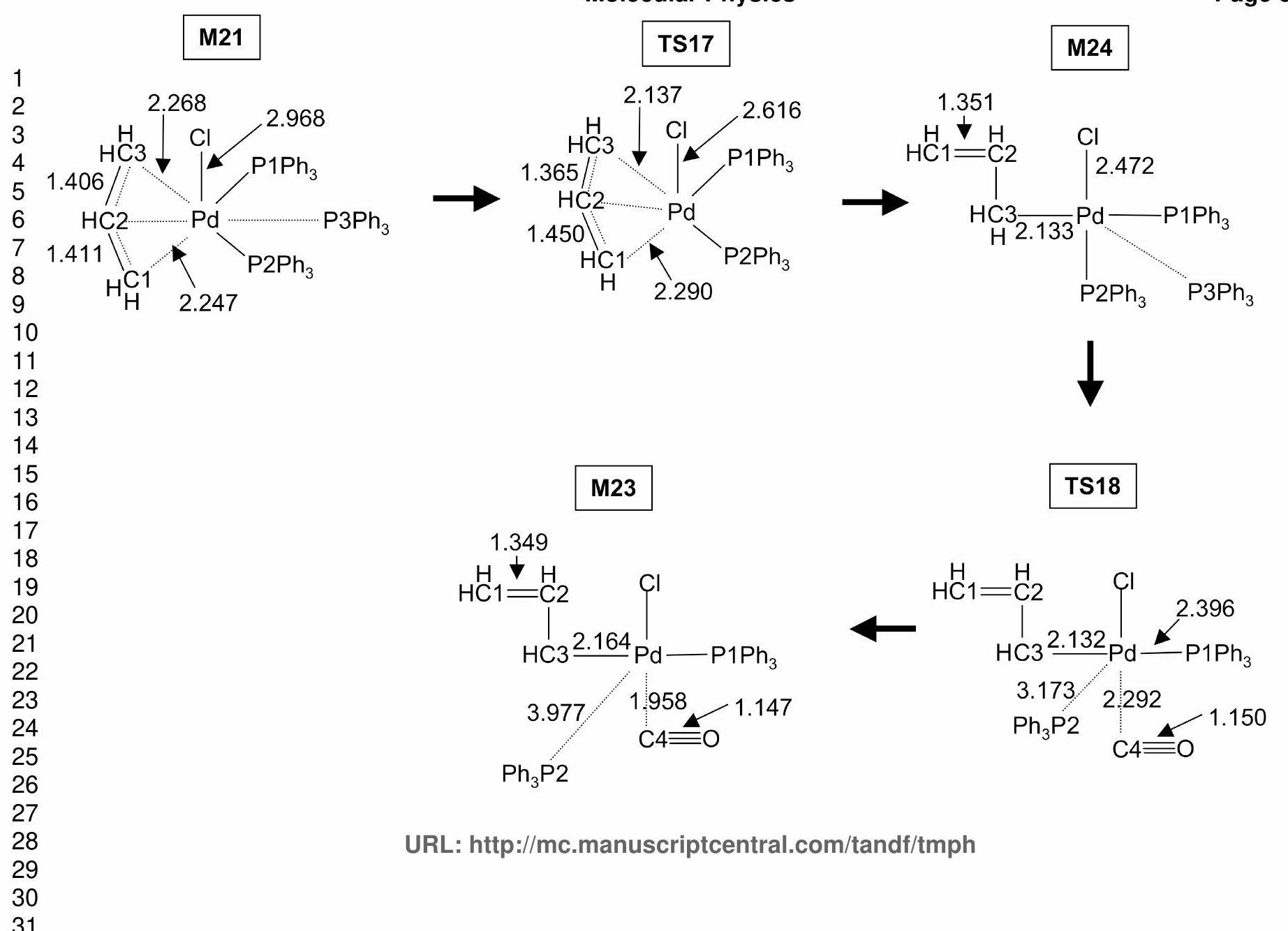




\section{Page 51 of 69}

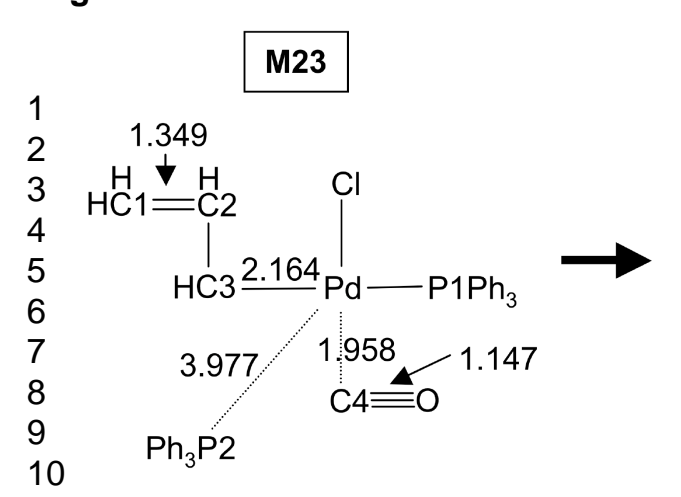

11

12

13

14

15

16

17

18

19

20

21

22

23

24

25

26

27

28
Molecular Physics
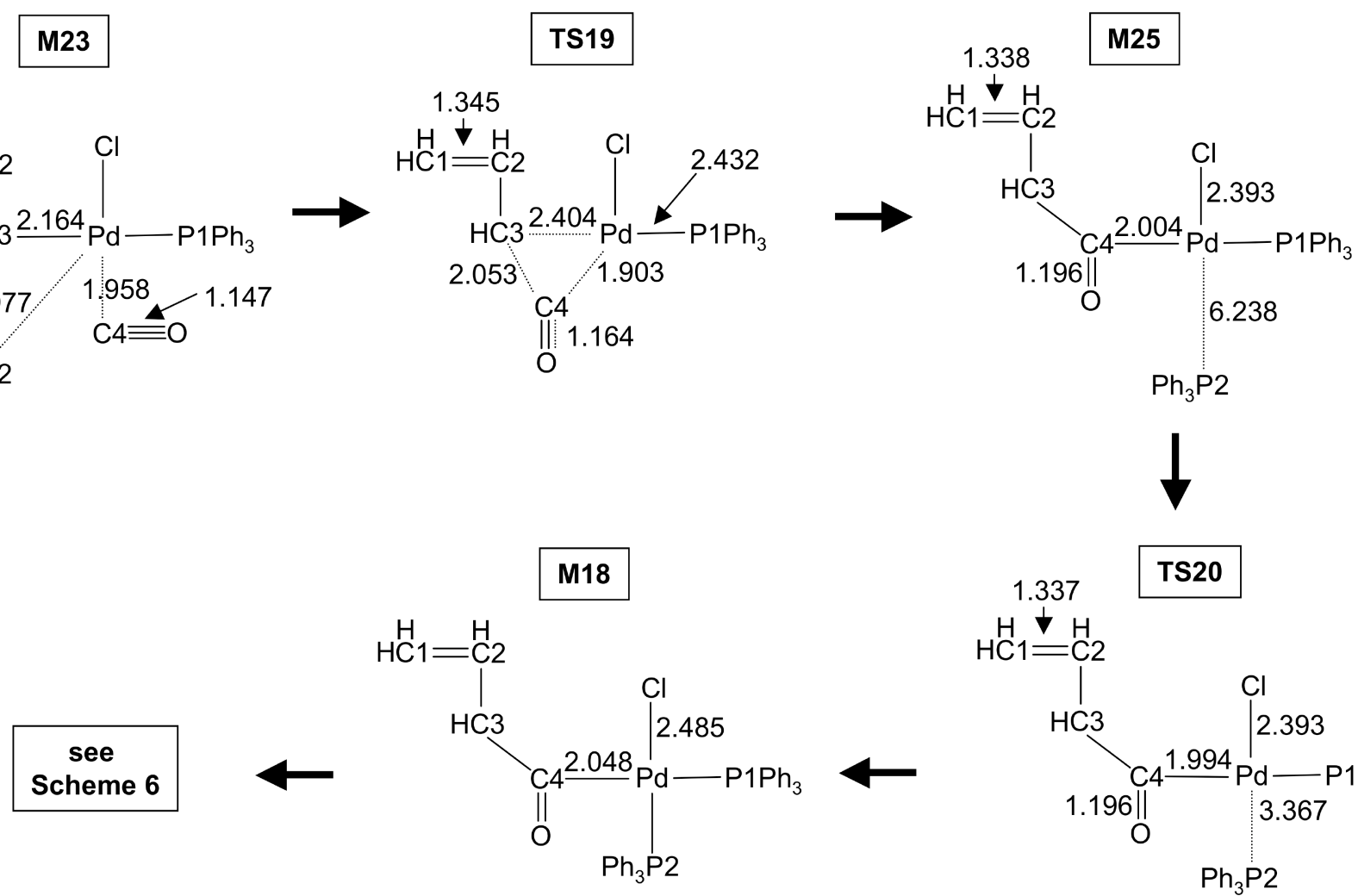

URL: http://mc.manuscriptcentral.com/tandf/tmph 


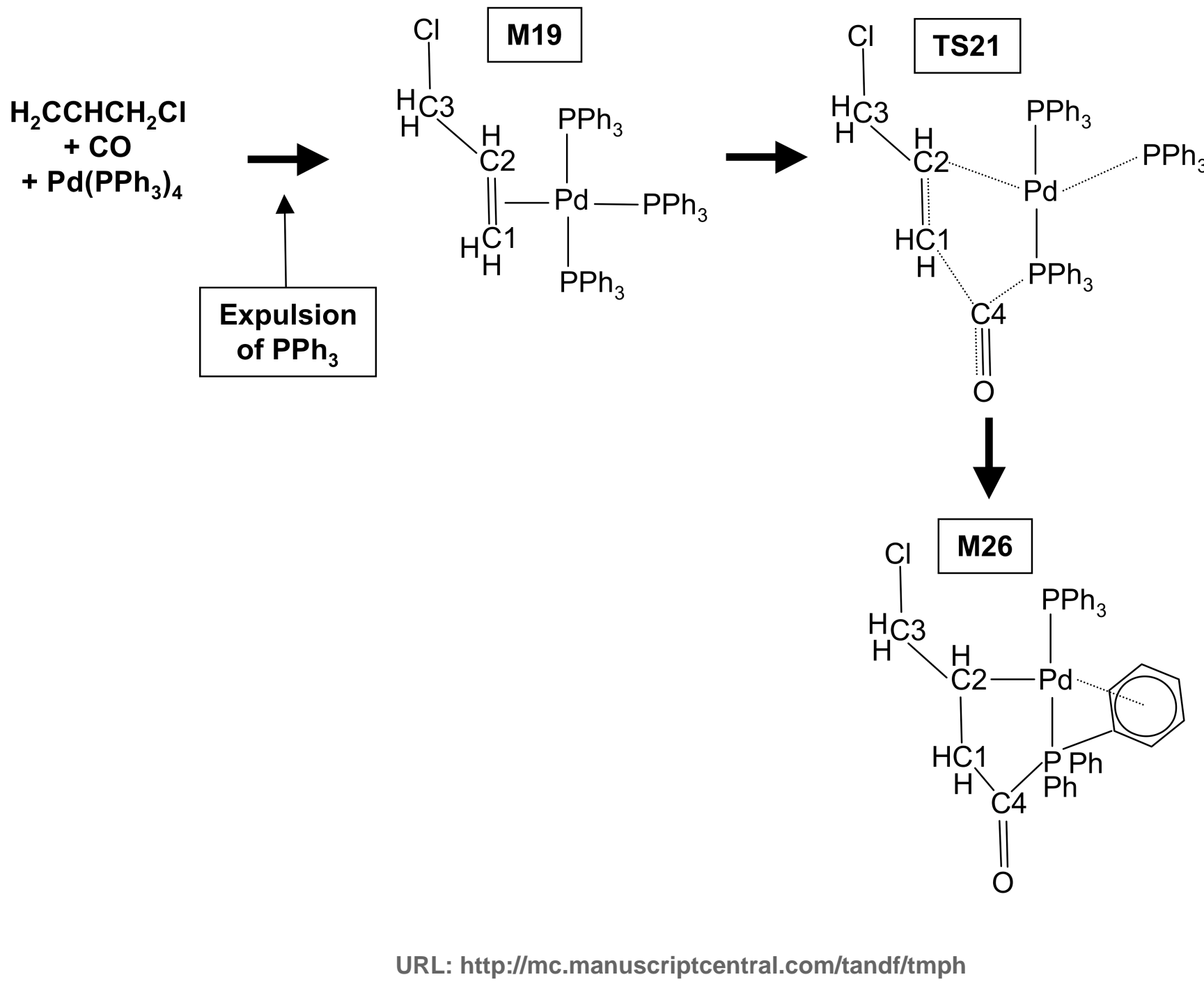


Table 1. Selected bond lengths ( $)$ ) for the critical points M0, M1, TS1, M2 and M3.

\begin{tabular}{llllllllll}
\hline & Pd-O1 & Pd-O2 & Pd-O3 & Pd-O4 & Pd-P1 & O1-H1 & Pd-P2 & O1-H2 \\
\hline M0 & 2.094 & 2.097 & 2.104 & 2.101 & $/$ & $/$ & $/$ & $/$ \\
M1 & 2.106 & 2.095 & 2.100 & 2.109 & 3.747 & 2.439 & $/$ & $/$ \\
TS1 & 2.251 & 2.053 & 2.163 & 2.081 & 3.072 & 2.440 & $/$ & $/$ \\
M2 & 3.088 & 2.042 & 2.239 & 2.119 & 2.304 & $/$ & $/$ & $/$ \\
M3 & 2.991 & 2.056 & 2.199 & 2.132 & 2.309 & $/$ & 4.110 & 2.292 \\
\hline
\end{tabular}


Table 2. Selected bond lengths ( $($ ) for the critical points TS2, M4 and M5

\begin{tabular}{lllllllll}
\hline & Pd-O1 & Pd-O2 & Pd-O3 & Pd-O4 & Pd-P1 & Pd-P2 & O1-P1 & O3-P1 \\
\hline TS2 & 3.048 & 2.109 & 2.377 & 2.125 & 2.296 & 3.040 & 4.087 & 3.927 \\
M4 & 3.005 & 2.073 & 3.181 & 2.082 & 2.396 & 2.412 & 3.427 & 4.376 \\
M5 & 3.137 & 2.084 & 3.047 & 2.064 & 2.410 & 2.410 & 2.884 & 3.862
\end{tabular}


Table 3. Selected bond lengths ( $⿱$ A) for the critical points TS3, M6, TS4 and M7

\begin{tabular}{llllllllllll} 
& Pd-O2 & Pd-O4 & O1-P1 & O3-P1 & Pd-P1 & Pd-P2 & P1-C1 & Pd-C1 & Pd-C2 & Pd-P3 \\
TS3 & 2.539 & 2.438 & 1.861 & 1.904 & 2.478 & 2.504 & 1.914 & 2.402 & 2.686 & $/$ \\
M6 & 3.743 & 5.134 & 1.857 & 1.863 & 5.134 & 2.355 & 1.820 & 2.310 & 2.397 & 4.743 \\
TS4 & 3.520 & 4.529 & 1.861 & 1.863 & 4.530 & 2.361 & 1.824 & 3.226 & 2.446 & 3.544 \\
M7 & / & / & 1.846 & 1.846 & $/$ & 2.365 & $/$ & $/$ & $/$ & 2.365 \\
\hline
\end{tabular}


Table 4. Selected bond lengths ( $\AA$ ) for the critical points M8, TS5, M9, M10, TS6 and M11.

\begin{tabular}{lllllll}
\hline & Pd-P2 & Pd-P3 & Pd-P4 & C3-H3 & Pd-P5 & C4-H4 \\
\hline M8 & 2.365 & 2.370 & 4.856 & 2.800 & $/$ & \\
TS5 & 2.368 & 2.371 & 3.970 & 3.180 & $/$ & \\
M9 & 2.431 & 2.424 & 2.416 & $/$ & $/$ & \\
M10 & 2.421 & 2.413 & 2.431 & $/$ & 5.161 & 2.811 \\
TS6 & 2.462 & 2.465 & 2.459 & $/$ & 3.447 & $/$ \\
M11 & 2.550 & 2.543 & 2.550 & $/$ & 2.535 &
\end{tabular}


Table 5. Selected bond lengths ( $)$ ) for the critical points TS7, M12 and TS8.

\begin{tabular}{llllllllll}
\hline & Pd-O1 & Pd-O2 & Pd-O3 & Pd-O4 & Pd-P1 & Pd-P2 & O2-P3 & O1-P3 & O4-P3 \\
\hline TS7 & 3.120 & 2.166 & 2.117 & 2.420 & 2.300 & 2.787 & $/$ & $/$ & $/$ \\
M12 & 2.949 & 2.118 & 2.119 & 2.967 & 2.327 & 2.330 & $/$ & $/$ & $/$ \\
TS8 & / & 2.649 & 2.252 & 3.573 & 2.513 & 2.366 & 2.130 & 2.572 & 2.941
\end{tabular}


Table 6. Selected bond lengths (Å) for the critical points M13, M14, TS9 and M15.

\begin{tabular}{llllllll}
\hline & P1-O1 & P1-O3 & P1-O5 & H1-O2 & H1-O5 & H2-O4 & H2-O5 \\
\hline M13 & 1.846 & 1.846 & $/$ & $/$ & 0.969 & $/$ & 0.969 \\
M14 & 1.876 & 1.827 & $/$ & 1.900 & 0.980 & $/$ & 0.968 \\
TS9 & 2,131 & 1.750 & 1.918 & 1.172 & 1.244 & 1.968 & 0.980 \\
M15 & 4.156 & 4.032 & 1.529 & 0.998 & 1.735 & 0.999 & 1.728
\end{tabular}


Table 7. Selected bond lengths ( $\AA$ ) for the critical points M16, TS10, M17, TS11, M18 and TS12.

\begin{tabular}{lllllllll}
\hline & Pd-P1 & Pd-P2 & Pd-P3 & Pd-Cl & C3-Cl & C4-Cl & Pd-C4 & C1-C4 \\
\hline M16 & 2.422 & 2.433 & 2.424 & 4.697 & 1.848 & $/$ & $/$ & $/$ \\
\hline TS10 & 2.430 & 2.453 & 2.438 & 8.422 & 1.842 & $/$ & 3.244 & $/$ \\
\hline M17 & 2.533 & 2.515 & 2.518 & 7.482 & 1.844 & $/$ & 2.015 & $/$ \\
TS11 & 2.535 & 2.508 & 2.626 & 4.654 & 2.392 & $/$ & 2.087 & 1.912 \\
M18 & 2.618 & 2.408 & 4.291 & 2.485 & $/$ & 2.860 & 2.048 & 1.541 \\
TS12 & 2.684 & 2.437 & 2.698 & 2.928 & $/$ & 1.998 & 2.384 & 1.530
\end{tabular}


Table 8. Selected bond lengths (Å) for the critical points M19, TS13, M20.

\begin{tabular}{llllllll}
\hline & Pd-C1 & Pd-C2 & Pd-P1 & Pd-P2 & Pd-P3 & C3-Cl & C1-C2 \\
\hline M19 & 2.386 & 2.443 & 2.492 & 2.506 & 2.622 & 1.880 & 1.373 \\
TS13 & 2.321 & 2.368 & 2.473 & 2.481 & 2.856 & 1.881 & 1.381 \\
M20 & 2.227 & 2.263 & 2.427 & 2.438 & 4.877 & 1.885 & 1.396
\end{tabular}


Table 9. Selected bond lengths (Å) for the critical points TS14, M21, TS15 and M22.

\begin{tabular}{llllllllll}
\hline & Pd-C1 & Pd-C2 & Pd-C3 & Pd-P1 & Pd-P2 & Pd-Cl & C3-Cl & C1-C2 & C2-C3 \\
\hline TS14 & 2.147 & 2.337 & 3.040 & 2.366 & 2.529 & 3.298 & 2.637 & 1.429 & 1.392 \\
M21 & 2.247 & 2.251 & 2,268 & 2.428 & 2.442 & 2.968 & $/$ & 1.411 & 1.406 \\
TS15 & 2.222 & 2.240 & 2.266 & 2.395 & 2.905 & 2.576 & $/$ & 1.412 & 1.405 \\
M22 & 2.242 & 2.225 & 2.201 & 2.404 & 4.540 & 2.440 & $/$ & 1.402 & 1.417
\end{tabular}


Table 10. Selected bond lengths (̊̊) for the critical points TS16, M23, TS17, M24 and TS18.

\begin{tabular}{llllllllll}
\hline & Pd-C1 & Pd-C3 & Pd-C4 & Pd-P1 & Pd-P2 & Pd-Cl & C4-C1 & C1-C2 & C2-C3 \\
\hline TS16 & 2.247 & 2.253 & 2.244 & 2.389 & 5.167 & 2.588 & 3.445 & 1.411 & 1.410 \\
M23 & $/$ & 2.164 & 1.958 & 2.504 & 3.977 & 2416 & 2.813 & 1.349 & 1.471 \\
TS17 & 2.890 & 2.137 & $/$ & 2.395 & 2.467 & 2.616 & $/$ & 1.365 & 1.450 \\
M24 & $/$ & 2.133 & $/$ & 2.375 & 2.568 & 2.472 & $/$ & 1.351 & 1.471 \\
TS18 & $/$ & 2.132 & 2.292 & 2.396 & 3.173 & 2.460 & 3.924 & 1.351 & 1.465
\end{tabular}


Table 11. Selected bond lengths ( $)$ ) for the critical points TS19, M25 and TS20

\begin{tabular}{llllllllll} 
Table 11. Selected bond lengths $(\AA)$ for the critical points TS19, M25 and TS20 \\
\hline & C4-O & Pd-C3 & Pd-C4 & Pd-P1 & Pd-P2 & Pd-Cl & C4-C1 & C1-C2 & C2-C3 \\
\hline TS19 & 1.164 & 2.404 & 1.903 & 2.387 & 5.763 & 2.432 & 2.053 & 1.345 & 1.478 \\
M25 & 1.196 & $/$ & 2.004 & 2.375 & 6.238 & 2.393 & 1.536 & 1.338 & 1.509 \\
TS20 & 1.196 & $/$ & 1.994 & 2.389 & 3.367 & 2.402 & 1.527 & 1.337 & 1.507
\end{tabular}


Supporting information file for the paper:

\title{
A Theoretical Investigation on the Oxidation States of Palladium Complexes and their Role in Carbonylation Reaction
}

\author{
Andrea Bottoni $^{(\mathrm{a})}$, Maria Angels Carvajal ${ }^{(\mathrm{b})}$,* Gian Pietro Miscione ${ }^{(\mathrm{a})}$,* \\ Juan J. Novoa ${ }^{(\mathrm{c})}$ \\ Email: gianpietro.miscione@unibo.it
}

\section{A. Full geometry optimizations with the DZVP basis set on all atoms}

To check the reliability of the approach using the DZVP basis set on all atoms except the phenyl rings (on which the $3-21 G^{*}$ basis set has been employed), we have carried out additional computations (with full geometry optimization) using the DZVP basis set on all atoms and we have compared the results to those obtained at the DZVP/3$21 \mathrm{G}^{*}$ level. The full geometry optimizations have been performed on the following critical points: M5, TS3, M6, TS4, M11, TS11, M20, TS14, corresponding to four key steps. We have found that the more accurate representation provides results (energetics and geometrical parameters) very similar to the DZVP/3-21G* basis.

We report here the schematic representation of the eight structures with the values of the most important geometry parameters obtained at the DZVP/3-21G* level and with the full DZVP basis set (values in italic and in brackets). The four activation energy barriers are also displayed: values obtained with the DZVP basis set are in italic and in brackets. 

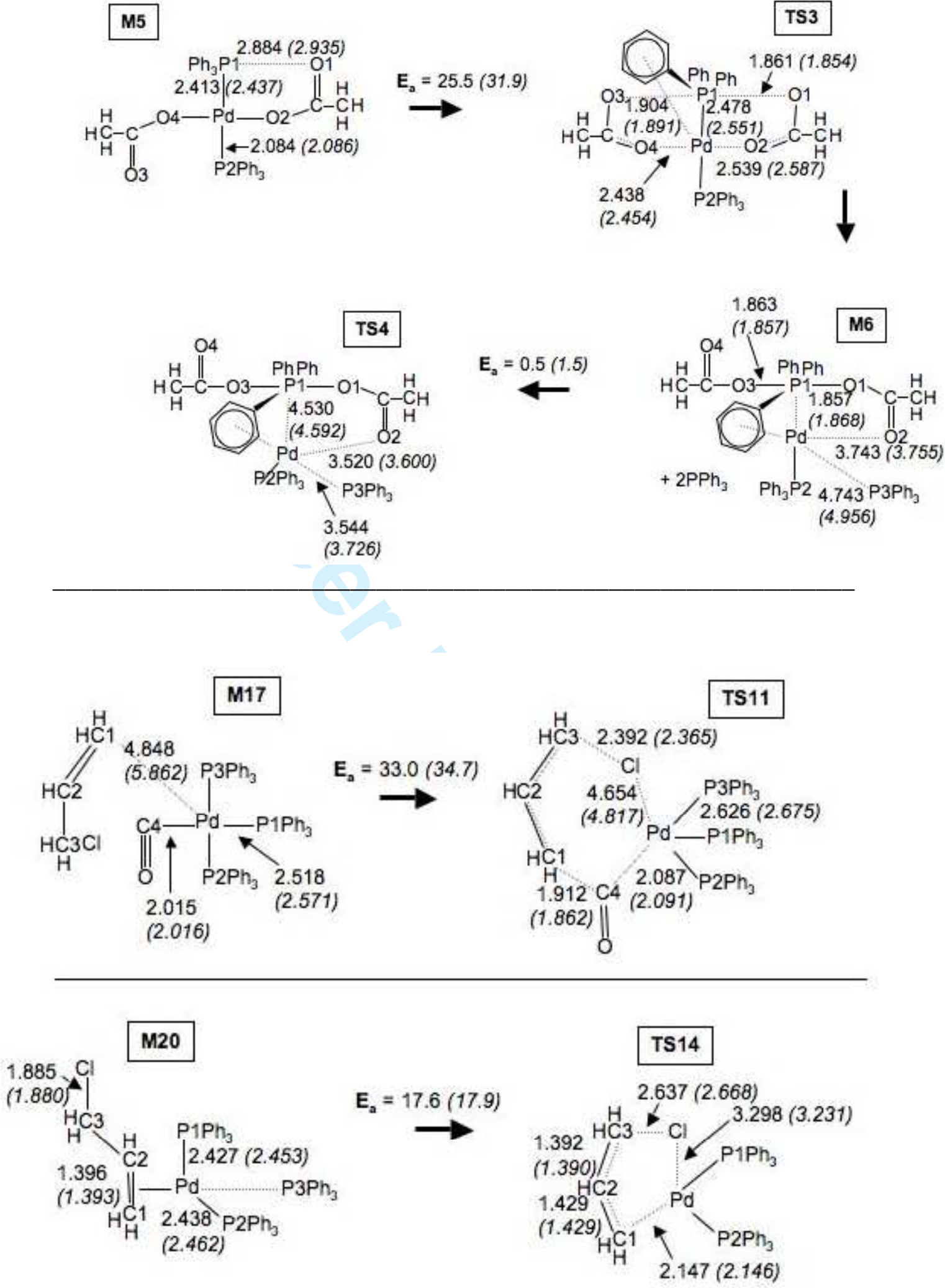


\section{B. IRC calculations}

A series of IRC computations has been carried out starting from the most important transition state structures in order to confirm the topology connections. The transition states for which the IRC calculations have been performed are the following: TS3 (toward reagents and products), TS4 (toward reagents), TS11 (toward reagents), TS19 (toward reagents and products). In several cases, as the transition state involves the displacement of a phosphine group from the metal, the IRC calculations cannot clearly indicate a complete separation of the two groups. As a matter of fact, once the Pd-P bond has slightly elongated in the first steps of the IRC calculation, the phosphine group tends to maintain a slight interaction with the metal and not to totally separate. However, even in these cases, the vibrational analysis undoubtedly shows that the normal mode with imaginary frequency involves the breaking of the Pd-P bond.

We report here the results of the IRC calculations as (i) schematic representation of the structure of the initial transition state, the last point of the IRC and the following intermediate on the PES; (ii) graphics in which the y axis represents the energy of the various IRC points (in $\mathrm{kcal} \mathrm{mol}^{-1}$, the energy of the transition state is the reference) and the $\mathrm{x}$ axis the net reaction coordinate.

TS3 toward M5 (reagents)

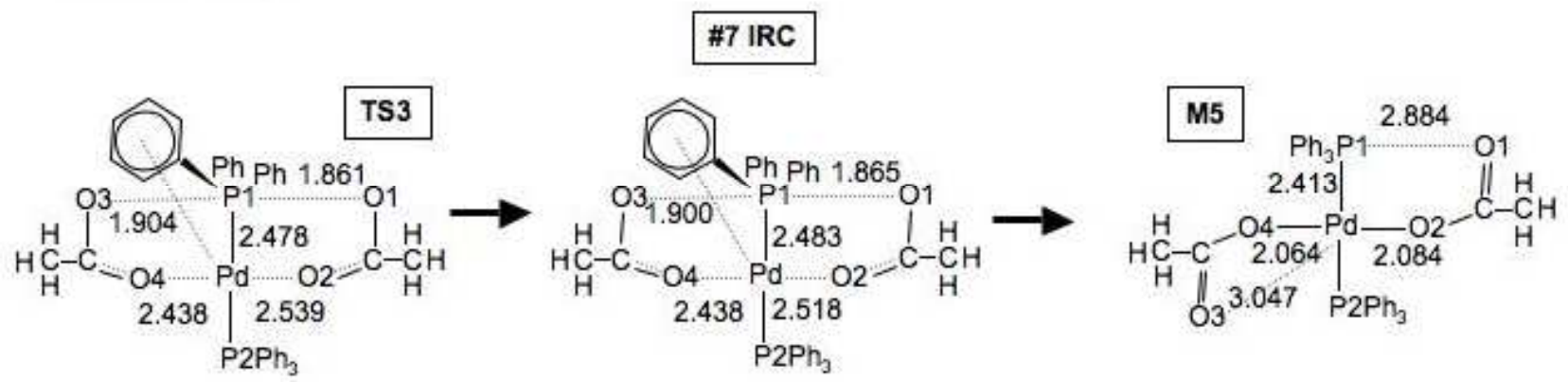




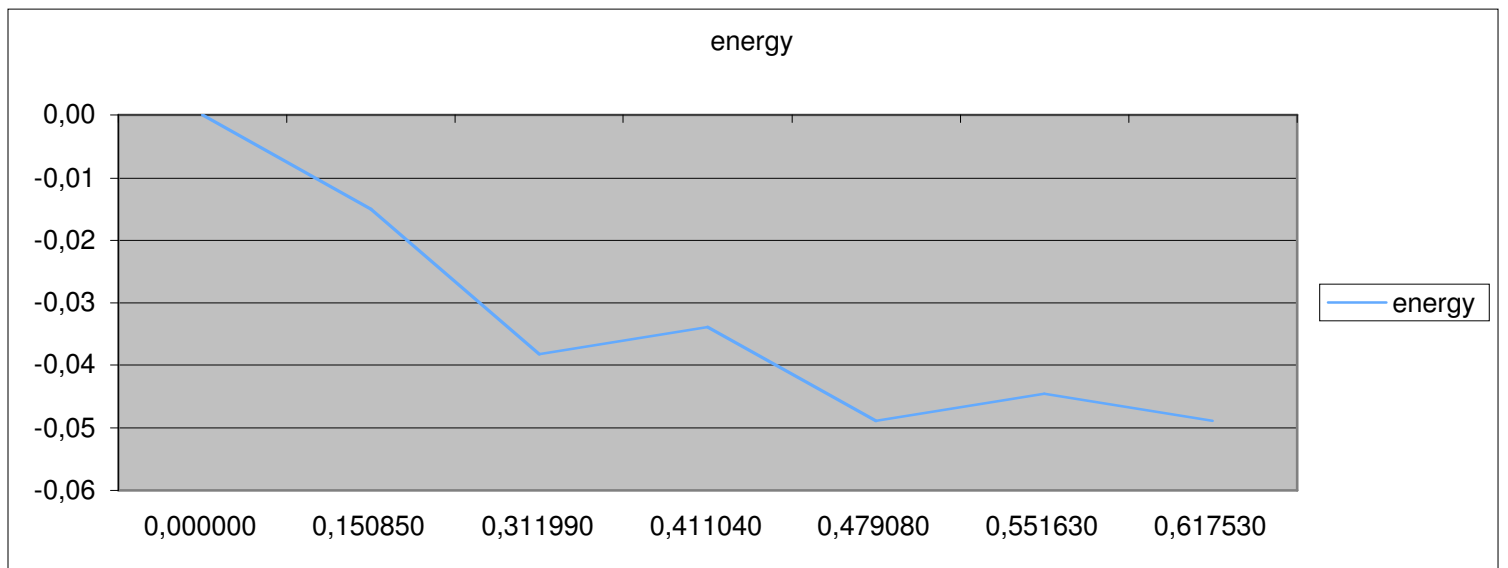

TS3 toward M6 (products)
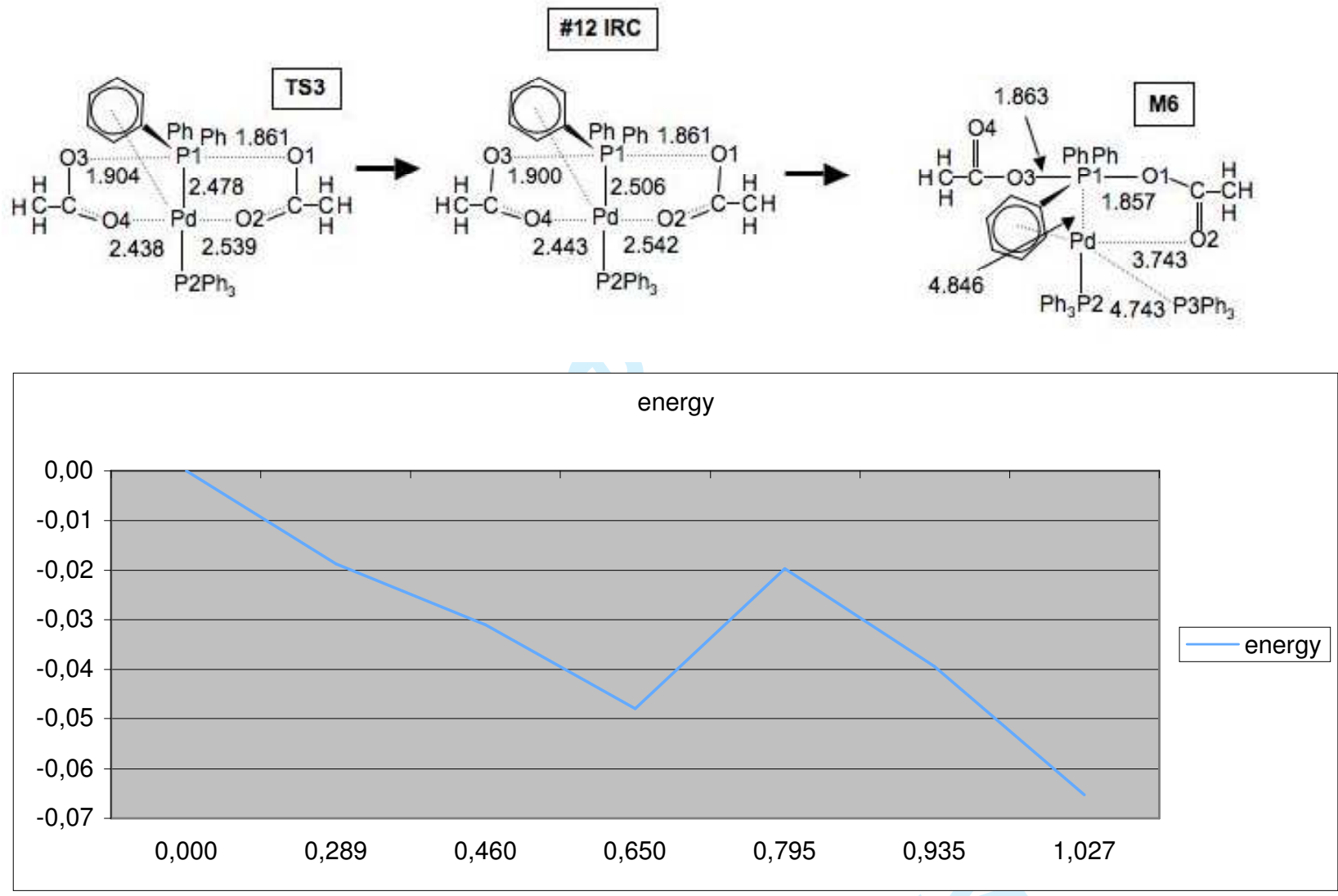

TS4 toward M6 (reagents) 

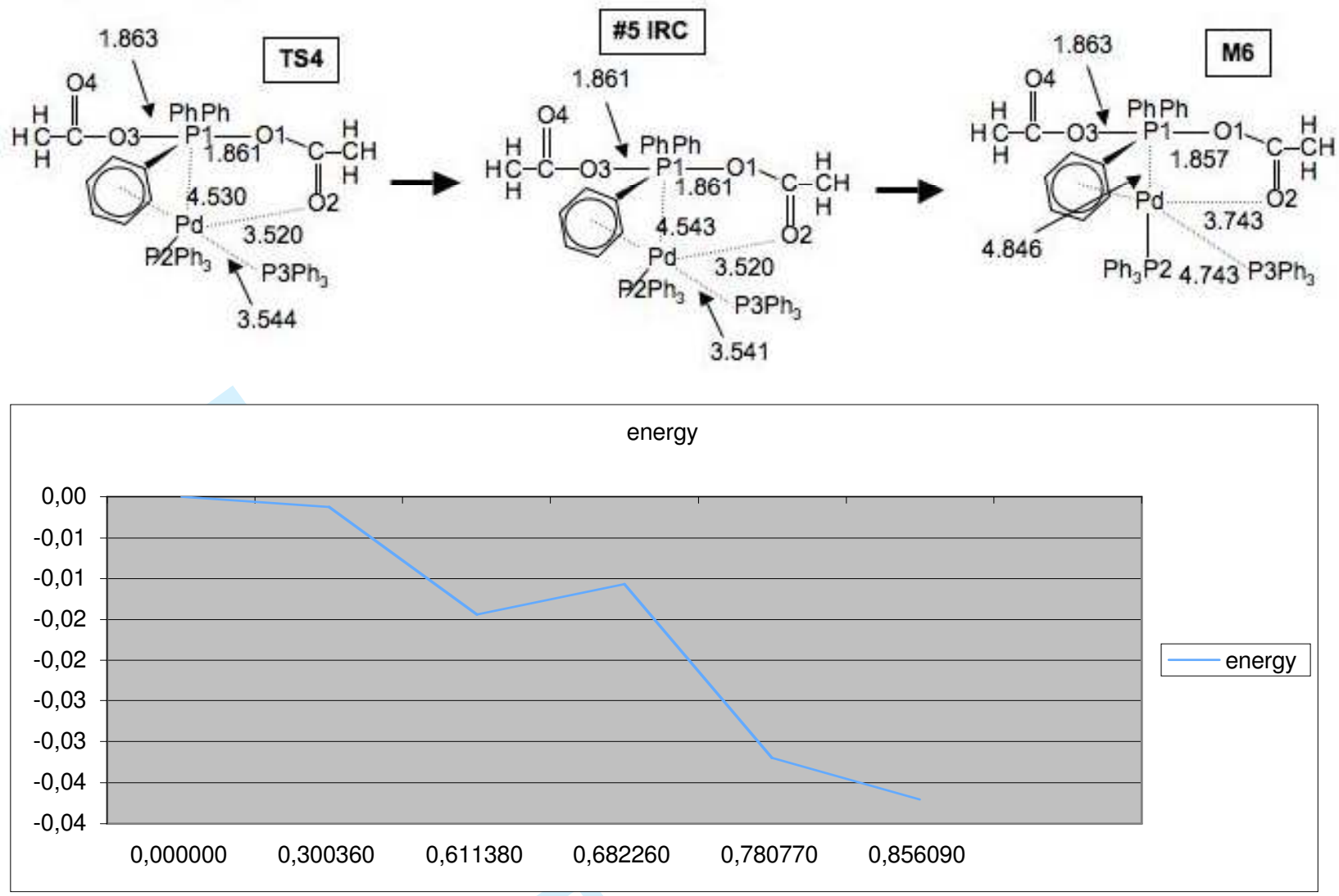

TS11 toward M17 (reagents)
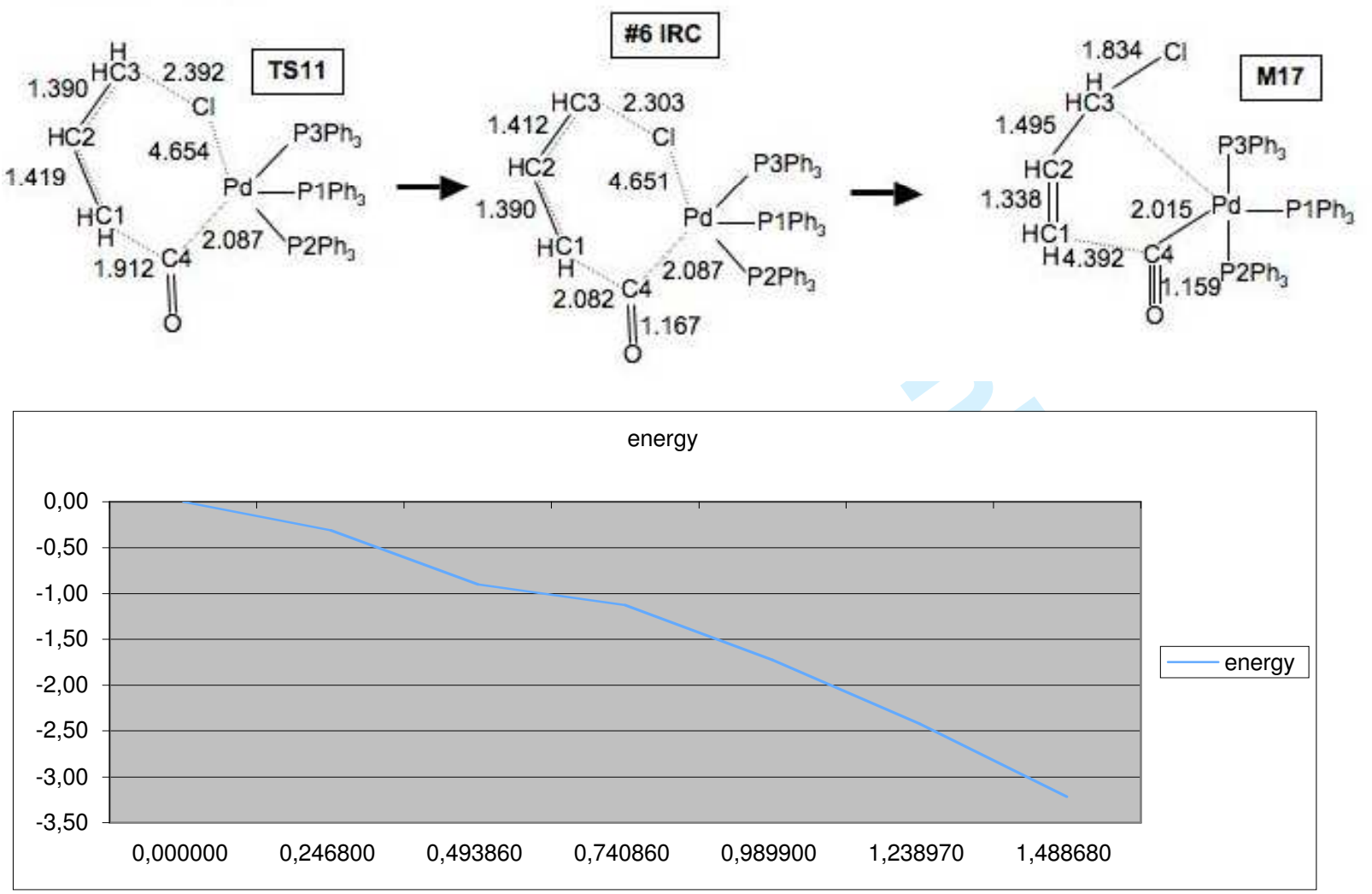

TS19 toward M23 (reagents) 

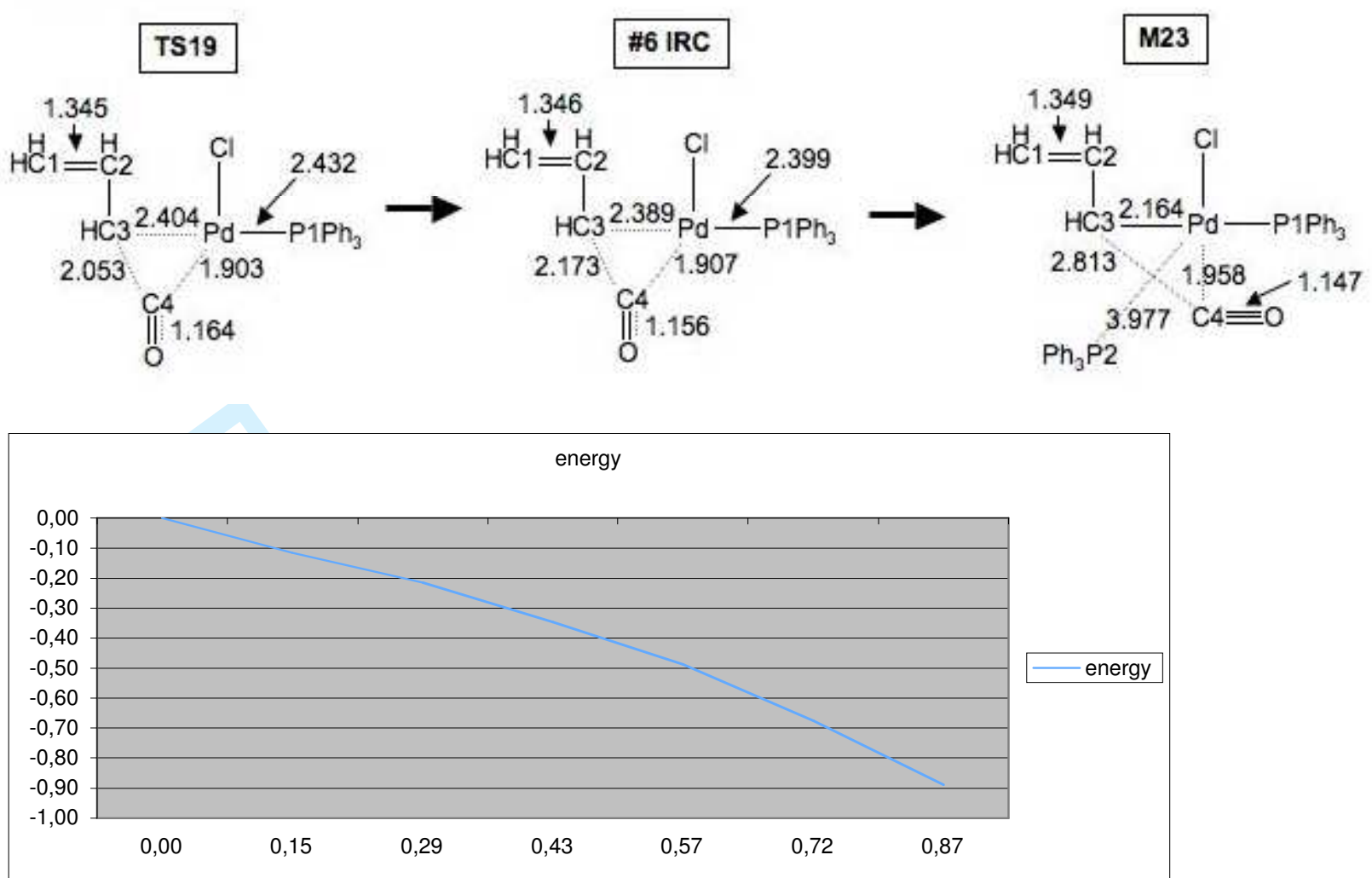

TS19 toward M25 (products)
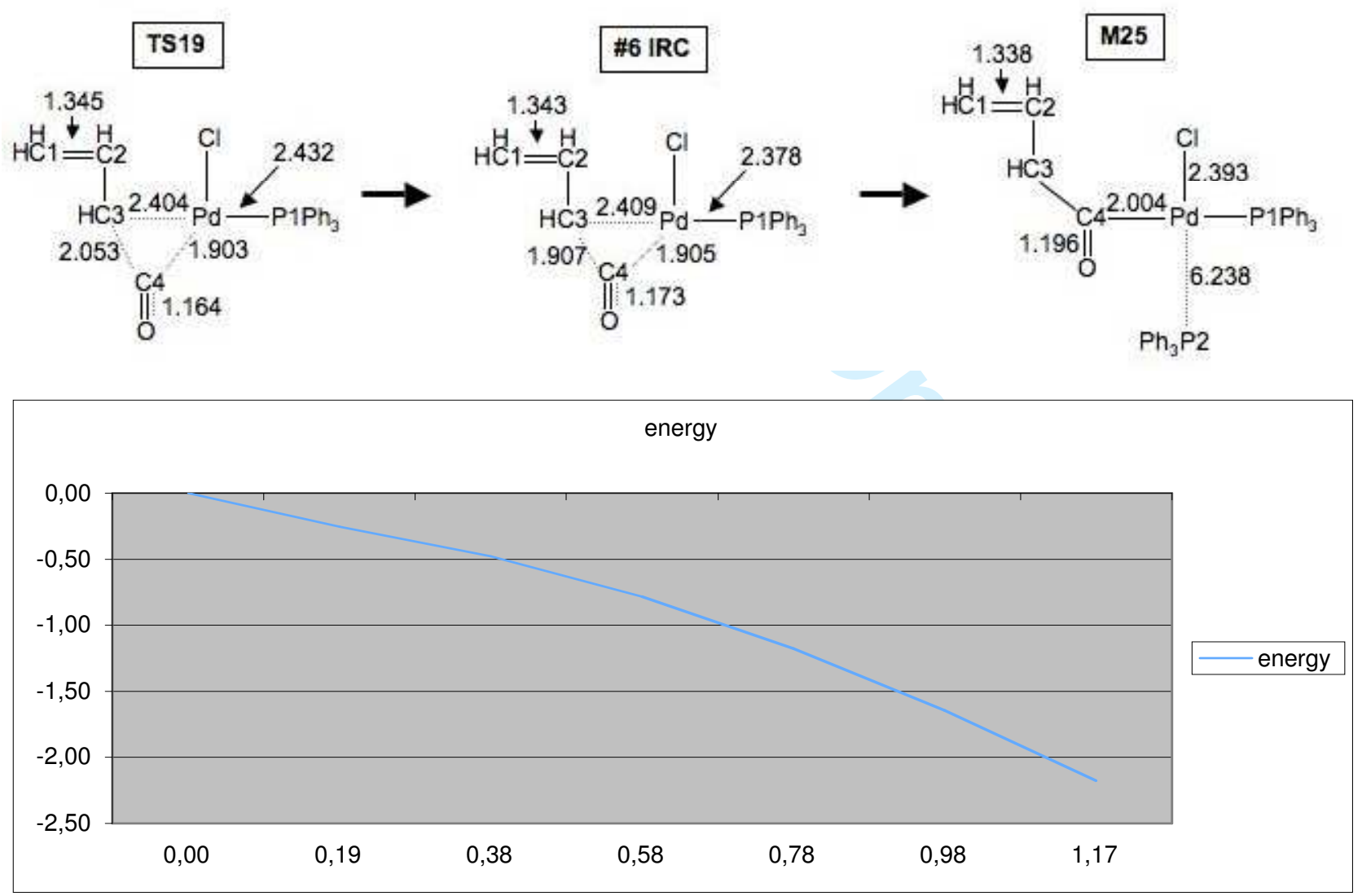